hep-ph/0206034

ROMA-1336/02

\title{
The silver channel at the Neutrino Factory
}

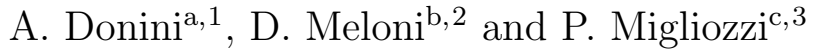 \\ a I.N.F.N., Sezione di Roma I and Dip. Fisica, Università di Roma "La Sapienza", \\ P.le A. Moro 2, I-00185, Rome, Italy \\ b Dip. Fisica, Università di Roma "La Sapienza" and I.N.F.N., Sezione di Roma I, \\ P.le A. Moro 2, I-00185, Rome, Italy \\ c I.N.F.N., Sezione di Napoli, Complesso Universitario di Monte Sant'Angelo, Via \\ Cintia ed. G, I-80126 Naples, Italy
}

\begin{abstract}
We notice that looking for $\nu_{e} \rightarrow \nu_{\tau}$ at the same time as $\nu_{e} \rightarrow \nu_{\mu}$ oscillations could significantly help to reduce the errors in the leptonic CP-violating phase $\delta$ measurement. We show how the $\nu_{e} \rightarrow \nu_{\mu}$ ("golden") and $\nu_{e} \rightarrow \nu_{\tau}$ ("silver") transitions observed at an OPERA-like 2 Kton lead-emulsion detector at $L=$ $732 \mathrm{Km}$, in combination with the $\nu_{e} \rightarrow \nu_{\mu}$ transitions observed at a $40 \mathrm{Kton}$ magnetized iron detector with a baseline of $L=3000 \mathrm{Km}$, strongly reduce the socalled $\left(\theta_{13}, \delta\right)$ ambiguity. We also show how a moderate increase in the OPERAlike detector mass (4 Kton instead of 2 Kton) completely eliminates the clone regions even for small values of $\theta_{13}$.
\end{abstract}

\footnotetext{
${ }^{1}$ andrea.donini@roma1.infn.it

${ }^{2}$ davide.meloni@roma1.infn.it

${ }^{3}$ pasquale.migliozzi@cern.ch
} 


\section{Introduction}

The present atmospheric [1]- [6] and solar [7]-[14] neutrino data are strongly supporting the hypothesis of neutrino oscillations [15-18 and can be easily accommodated in a three family mixing scenario.

Let the Pontecorvo-Maki-Nakagawa-Sakata $U_{P M N S}$ matrix be the leptonic analogue of the hadronic Cabibbo-Kobayashi-Maskawa (CKM) mixing matrix in its most conventional parametrization [19]:

$$
U_{P M N S}=\left(\begin{array}{ccc}
1 & 0 & 0 \\
0 & c_{23} & s_{23} \\
0 & -s_{23} & c_{23}
\end{array}\right)\left(\begin{array}{ccc}
c_{13} & 0 & s_{13} e^{i \delta} \\
0 & 1 & 0 \\
-s_{13} e^{-i \delta} & 0 & c_{13}
\end{array}\right)\left(\begin{array}{ccc}
c_{12} & s_{12} & 0 \\
-s_{12} & c_{12} & 0 \\
0 & 0 & 1
\end{array}\right)
$$

with the short-form notation $s_{i j} \equiv \sin \theta_{i j}, c_{i j} \equiv \cos \theta_{i j}$. Oscillation experiments are sensitive to the two neutrino mass differences $\Delta m_{12}^{2}, \Delta m_{23}^{2}$ and to the four parameters in the mixing matrix: three angles and the Dirac CP-violating phase, $\delta$.

In particular, data on atmospheric neutrinos are interpreted as oscillations of muon neutrinos into neutrinos that are not $\nu_{e}$ 's, with a mass gap that we denote by $\Delta m_{23}^{2}$. The corresponding mixing angle is close to maximal, $\sin ^{2} 2 \theta_{23}>0.8$, and $\left|\Delta m_{23}^{2}\right|$ is in the range 1.8 to $4 \times 10^{-3} \mathrm{eV}^{2}$ [20].

The recent SNO results for solar neutrinos [12]-14 favour the LMA-MSW [21] solution of the solar neutrino deficit with $\nu_{e}$ oscillations into active $\left(\nu_{\mu}, \nu_{\tau}\right)$ neutrino states. The corresponding squared mass difference, that in this parametrization should be identified with $\Delta m_{12}^{2}$, is $\sim 10^{-5}-10^{-4} \mathrm{eV}^{2}$. Comprehensive analyses of the solar neutrino data, however, do not exclude the LOW-MSW solution [22]-[25], with $\Delta m_{12}^{2} \sim$ $10^{-7} \mathrm{eV}^{2}$. In both cases, the corresponding mixing angle $\left(\theta_{12}\right)$ is large (albeit not maximal).

Finally, the LSND data [26, 27] would indicate a $\nu_{\mu} \rightarrow \nu_{e}$ oscillation with a third, very distinct, neutrino mass difference: $\Delta m_{L S N D}^{2} \sim 0.3-6 \mathrm{eV}^{2}$. The LSND evidence in favour of neutrino oscillation has not been confirmed by other experiments so far [28]; the MiniBooNE experiment [29] will be able to do it in the near future [30]. In the absence of an independent confirmation of the LSND evidence, we restrict ourselves to the three neutrino mixing scenario (the impact of a Neutrino Factory in the case of four neutrino mixing has been discussed in full detail in [31]-33]).

These oscillation signals will be confirmed in ongoing and planned atmospheric and solar neutrino experiments, as well as in long baseline ones, with the latter being free of model-dependent estimations of neutrino fluxes. There is a strong case for going further in the fundamental quest of the neutrino masses and mixing angles, as a necessary step to unravel the fundamental new scale(s) behind neutrino oscillations. In particular, it is possible that in ten years from now no information whatsoever will be at hand regarding the $\theta_{13}$ angle (the key between the atmospheric and solar neutrino realms, for 
which the present bound is $\sin ^{2}\left(2 \theta_{13}\right) \leq 1 \times 10^{-1}$, [34]) and the leptonic CP violating phase $\delta$.

An experimental set-up with the ambitious goal of precision measurement of the whole three-neutrino mixing parameter space is under study. This experimental programme consists of the development of a "Neutrino Factory" (high-energy muons decaying in the straight section of a storage ring and producing a very pure and intense neutrino beam, [35, 36]) and of suitably optimized detectors located far away from the neutrino source. The effort to prepare such very long baseline neutrino experiments will require a time period covering this and the beginning of the following decade. It is therefore of interest to look for the optimal conceivable factory-detectors combination. One of its main goals would be the discovery of leptonic CP violation and, possibly, its study [37- 40]. Previous analyses [41]- 413] on the foreseeable outcome of experiments at a Neutrino Factory have shown that the determination of the two still unknown parameters in the three-neutrino mixing matrix, $\theta_{13}$ and $\delta$, will be possible (if the LMA-MSW solution of the solar neutrino deficit is confirmed). The most sensitive method to study these topics is to measure the transition probabilities involving $\nu_{e}$ and $\bar{\nu}_{e}$, in particular $\nu_{e}\left(\bar{\nu}_{e}\right) \rightarrow \nu_{\mu}\left(\bar{\nu}_{\mu}\right)$. This is what is called the "golden measurement at the neutrino factory". Such a facility is indeed unique in providing high energy and intense $\nu_{e}\left(\bar{\nu}_{e}\right)$ beams. Since these beams contain no $\bar{\nu}_{\mu}\left(\nu_{\mu}\right)$, the transitions of interest can be measured by searching for "wrong-sign" muons: negative (positive) muons appearing in a massive detector with good muon charge identification capabilities [40].

An incredible amount of work has been devoted to this topic in the last few years: we refer the interested reader to [44]-[54] and to the refs. therein for an overview of the status-of-the-art in all its different aspects; we address to [55]- [60] and refs. therein for a comparison of the physics reach of a conventional (super)beam and of a Neutrino Factory; eventually, we point out that in [61] the idea of a $\bar{\nu}_{e}$ beam originating from $\beta$-decay (the so-called " $\beta$-beam"), was advanced: it appears that the physics reach of such a beam is complementary to that of a conventional superbeam [62].

In [63] it has been noticed that the probability $P_{\nu_{\alpha} \rightarrow \nu_{\beta}}\left(\bar{\theta}_{13}, \bar{\delta}, E_{\nu}\right)$ for neutrinos at a fixed energy (and for a given baseline) computed for a given theoretical input pair $\left(\bar{\theta}_{13}, \bar{\delta}\right)$ defines a continuous equiprobability curve in the $\left(\theta_{13}, \delta\right)$ plane. Therefore, for a fixed energy, a continuum of solutions reproduce the input probability. A second equiprobability curve is defined in this plane by the probability for antineutrinos at the same energy and with the same input parameters, $P_{\bar{\nu}_{\alpha} \rightarrow \bar{\nu}_{\beta}}\left(\bar{\theta}_{13}, \bar{\delta}, E_{\nu}\right)$. The two equiprobability curves have, quite generally, two intersection points: the first of them at $\left(\bar{\theta}_{13}, \bar{\delta}\right)$, the second at a different point $\left(\tilde{\theta}_{13}, \tilde{\delta}\right)$. It is the intersection of equiprobability curves from the neutrinos and antineutrinos that resolves the continuum degeneracy of solutions in the $\left(\theta_{13}, \delta\right)$ plane, restricting the allowed values for $\theta_{13}$ and $\delta$ to the two regions around $\left(\bar{\theta}_{13}, \bar{\delta}\right)$ and $\left(\tilde{\theta}_{13}, \tilde{\delta}\right)$. This second intersection, however, introduces an ambiguity in the measurement of the physical values of $\theta_{13}$ and $\delta$. Different proposals have been suggested to solve this ambiguity: in [63] the ambiguity is solved by fitting at two different baselines at the same time; another possibility is an increase in the 
energy resolution of the detector, [46, 50, 51]; see also [64].

New degeneracies have later been noticed [65], resulting from our ignorance of the sign of the $\Delta m_{a t m}^{2}$ squared mass difference (by the time the Neutrino Factory will be operational) and from the approximate $\left[\theta_{23}, \pi / 2-\theta_{23}\right]$ symmetry for the atmospheric angle.

In the first part of the paper we describe how the $\left(\theta_{13}, \delta\right)$ ambiguity arises in $\nu_{e} \rightarrow \nu_{\mu}$ oscillation due to the equiprobability curves in the $\left(\theta_{13}, \delta\right)$ plane at fixed neutrino energy. We then extend our analysis showing how the same phenomenon can be observed in a real experiment: equal-number-of-events (ENE) curves for any given neutrino energy bin appears and their intersections in the $\left(\theta_{13}, \delta\right)$ plane explain how and where do "clone" regions arise. Our analysis is afterwards compared with the results of simulations with a realistic magnetized iron detector (studied in [66]).

We then propose to reduce the continuum degeneracy and to resolve the last ambiguity using two baselines with detectors of different design. We notice that muons proceeding from $\tau$ decay when $\tau$ 's are produced via a $\nu_{e} \rightarrow \nu_{\tau}$ transition show a different $\left(\theta_{13}, \delta\right)$ correlation from those coming from $\nu_{e} \rightarrow \nu_{\mu}$ (first considered in [40]). By using a near lead-emulsion detector, capable of the $\tau$-decay vertex recognition, we can therefore use the complementarity of the information from $\nu_{e} \rightarrow \nu_{\tau}$ and from $\nu_{e} \rightarrow \nu_{\mu}$ to solve the $\left(\theta_{13}, \delta\right)$ ambiguity. We find that the combination of the near emulsion detector and of a massive magnetized iron detector at $L=3000 \mathrm{Km}$ could indeed help to achieve a good resolution in the $\left(\theta_{13}, \delta\right)$ plane.

In this paper we restrict ourselves to the $\left(\theta_{13}, \delta\right)$ ambiguity, by fixing $\theta_{23}=45^{\circ}$ and by choosing a given sign for $\Delta m_{a t m}^{2}$ (in the hypothesis that more information on the three neutrino spectrum will be available by the time the Neutrino Factory will be operational). However, we found that the core of our results do not depend on the sign of $\Delta m_{a t m}^{2}$ : indeed, we have been carrying on simulations with the opposite sign, with similar results (i.e., we still observe how "clone" regions disappear due to the two-detector types combination; notice, however, that the location of the "clone" regions and all the details of the simulation do depend on the sign of $\Delta m_{a t m}^{2}$ ). We remind that, if $\theta_{13}$ is not extremely small, the combined measurement of $\nu_{e} \rightarrow \nu_{\mu}$ and $\nu_{e} \rightarrow \nu_{\tau}$ transitions could significantly help in solving the $\left[\theta_{23}, \pi / 2-\theta_{23}\right]$ ambiguity.

In Section 2 we present our analysis of the $\nu_{e} \rightarrow \nu_{\mu}$ equiprobability curves in the $\left(\theta_{13}, \delta\right)$ plane; in Section 3 we introduce the corresponding equal-number-of-events (ENE) curves; in Section 4 we present a similar analysis for the $\nu_{e} \rightarrow \nu_{\tau}$ oscillation probability and study the impact of "silver" wrong-sign muon events; in Section 5 we show our results for the combination of a near $(L=732 \mathrm{Km})$ OPERA-like detector and of a $L=3000 \mathrm{Km}$ magnetized iron detector; in Section 6 we eventually draw our conclusions. In Appendix $\mathrm{A}$ a perturbative expansion in $\Delta \theta$ of the formulae of Sect. 2 is presented; in Appendix B we report some useful formulae for $\tau$ CC-interaction and decay. 


\section{$2 \quad \nu_{e} \rightarrow \nu_{\mu}$ equiprobability curves in the $\left(\theta_{13}, \delta\right)$ plane}

We consider the $\nu_{e} \rightarrow \nu_{\mu}$ transition, first. This channel has been shown to be the optimal one to measure simultaneously $\theta_{13}$ and $\delta$ at the Neutrino Factory in the context of three-family mixing, through the appearance of "wrong-sign" muons in the detector, [40]. It therefore deserves the nickname of "golden channel".

Following eq. (1) of [63] we get for the transition probability at second order in perturbation theory in $\theta_{13}, \Delta_{\odot} / \Delta_{a t m}, \Delta_{\odot} / A$ and $\Delta_{\odot} L$ (see also [67-69]),

$$
P_{e \mu}^{ \pm}\left(\bar{\theta}_{13}, \bar{\delta}\right)=X_{ \pm} \sin ^{2}\left(2 \bar{\theta}_{13}\right)+Y_{ \pm} \cos \left(\bar{\theta}_{13}\right) \sin \left(2 \bar{\theta}_{13}\right) \cos \left( \pm \bar{\delta}-\frac{\Delta_{a t m} L}{2}\right)+Z
$$

where \pm refers to neutrinos and antineutrinos, respectively, and

$$
\left\{\begin{array}{l}
X_{ \pm}=\sin ^{2}\left(\theta_{23}\right)\left(\frac{\Delta_{a t m}}{B_{\mp}}\right)^{2} \sin ^{2}\left(\frac{B_{\mp} L}{2}\right) \\
Y_{ \pm}=\sin \left(2 \theta_{12}\right) \sin \left(2 \theta_{23}\right)\left(\frac{\Delta_{\odot}}{A}\right)\left(\frac{\Delta_{a t m}}{B_{\mp}}\right) \sin \left(\frac{A L}{2}\right) \sin \left(\frac{B_{\mp} L}{2}\right) \\
Z=\cos ^{2}\left(\theta_{23}\right) \sin ^{2}\left(2 \theta_{12}\right)\left(\frac{\Delta_{\odot}}{A}\right)^{2} \sin ^{2}\left(\frac{A L}{2}\right)
\end{array}\right.
$$

with $Z=Z_{+}=Z_{-}$. In these formulae, $A=\sqrt{2} G_{F} n_{e}$ (expressed in $\mathrm{eV}^{2} / \mathrm{GeV}$ ) and $B_{\mp}=\left|A \mp \Delta_{a t m}\right|$ (with $\mp$ referring to neutrinos and antineutrinos, respectively). Finally, $\Delta_{a t m}=\Delta m_{a t m}^{2} / 2 E_{\nu}$ and $\Delta_{\odot}=\Delta m_{\odot}^{2} / 2 E_{\nu}$.

The parameters $\bar{\theta}_{13}$ and $\bar{\delta}$ are the physical parameters that must be reconstructed by fitting the experimental data with the theoretical formula for oscillations in matter. In what follows, the other parameters have been considered as fixed quantities, supposed to be known by the time when the Neutrino Factory will be operational. In particular, in the solar sector we fixed $\theta_{12}=33^{\circ}$ and $\Delta m_{\odot}^{2}=1.0 \times 10^{-4} \mathrm{eV}^{2}$ [22]-25], corresponding to the LMA region of the solar neutrino problem (accordingly to the recent SNO results [13, 14]); in the atmospheric sector, $\theta_{23}=45^{\circ}$ and $\Delta m_{\text {atm }}^{2}=2.9 \times 10^{-3} \mathrm{eV}^{2}$ [20] (notice that for $\theta_{23}=45^{\circ}$ the $\left[\theta_{23}, \pi / 2-\theta_{23}\right]$ ambiguity [65 is absent). Finally, we considered a fixed value for the matter parameter, $A=1.1 \times 10^{-4} \mathrm{eV}^{2} / \mathrm{GeV}$ for $L<4000 \mathrm{~km}$ and $A=1.5 \times 10^{-4} \mathrm{eV}^{2} / \mathrm{GeV}$ for $L>4000 \mathrm{~km}$, obtained by using the average matter density alongside the path for the chosen distance computed with the Preliminary Earth Model [70. For simplicity, we have not included errors on these parameters.

Eq. (1) leads to an equiprobability curve in the plane $\left(\theta_{13}, \delta\right)$ for neutrinos and antineutrinos of a given energy:

$$
P_{e \mu}^{ \pm}\left(\bar{\theta}_{13}, \bar{\delta}\right)=P_{e \mu}^{ \pm}\left(\theta_{13}, \delta\right)
$$

\footnotetext{
${ }^{4}$ It has been shown in 63 that the inclusion of the foreseeable uncertainties on these parameters does not modify the results on the $\theta_{13}$ and $\delta$ measurements in a relevant manner.
} 
We can solve eq. (3) for $\delta$ :

$$
\cos \left( \pm \delta-\frac{\Delta_{a t m} L}{2}\right)=\frac{\bar{P}_{e \mu}^{ \pm}-X_{ \pm} \sin ^{2}\left(2 \theta_{13}\right)-Z}{Y_{ \pm} \cos \left(\theta_{13}\right) \sin \left(2 \theta_{13}\right)} .
$$

It is useful to introduce the following functions:

$$
\left\{\begin{array}{l}
f\left(\theta_{13}, \bar{\theta}_{13}\right)=\frac{\sin ^{2}\left(2 \bar{\theta}_{13}\right)-\sin ^{2}\left(2 \theta_{13}\right)}{\cos \theta_{13} \sin \left(2 \theta_{13}\right)} \\
g\left(\theta_{13}, \bar{\theta}_{13}\right)=\frac{\cos \bar{\theta}_{13} \sin \left(2 \bar{\theta}_{13}\right)}{\cos \theta_{13} \sin \left(2 \theta_{13}\right)}
\end{array}\right.
$$

with the obvious limit $f\left(\bar{\theta}_{13}, \bar{\theta}_{13}\right)=0$ and $g\left(\bar{\theta}_{13}, \bar{\theta}_{13}\right)=1$.

Eq. (₫) can then be written as:

$$
\cos \left( \pm \delta-\frac{\Delta_{a t m} L}{2}\right)=R_{ \pm} f\left(\theta_{13}, \bar{\theta}_{13}\right)+\cos \left( \pm \bar{\delta}-\frac{\Delta_{a t m} L}{2}\right) g\left(\theta_{13}, \bar{\theta}_{13}\right)
$$

Eq. (6) is particularly illuminating: it describes a family of two branches curves in the plane $\left(\theta_{13}, \delta\right)$ for the neutrinos and a second family of two branches curves for the antineutrinos. The dependence on the neutrino energy resides in $\Delta_{a t m}$ and in the ratio $R_{ \pm}=X_{ \pm} / Y_{ \pm}$, whereas the dependence on the angle is factorized in the two $\theta_{13}$-dependent functions $f$ and $g$.

It is helpful to introduce the parameter $\Delta \theta$ :

$$
\theta_{13}=\bar{\theta}_{13}+\Delta \theta
$$

constrained by the bound

$$
\left|\cos \left( \pm \delta-\frac{\Delta_{a t m} L}{2}\right)\right| \leq 1
$$

The allowed region for $\Delta \theta$ depend on the input parameters $\left(\bar{\theta}_{13}, \bar{\delta}\right)$, on the neutrino energy and on the baseline. In Fig. 1, we compute the allowed values of $\Delta \theta$ as a function of $\bar{\theta}_{13}\left(\bar{\theta}_{13} \in\left[0^{\circ}, 13^{\circ}\right]\right)$ at three different baselines, $L=732,3000$ and 7332 $\mathrm{Km}$, for $\bar{\delta}=0$ and $E_{\nu}=38 \mathrm{GeV}$, by numerically solving eq. (7).

It may be noticed that, for (almost) every value of $\bar{\theta}_{13}$ in the considered range, two different regions of allowed values for $\Delta \theta$ exist. The first region corresponds to $\Delta \theta \simeq 0$, whereas the second corresponds to large negative values for $\Delta \theta$. In this region, $\theta_{13}$ is negative: this region is therefore unphysical when the sign of the mass differences and of the various angles are defined in an appropriate way [52]. We concentrate hereafter on the tiny region around $\Delta \theta \simeq 0$.

In Fig. 2 we present the equiprobability curves for $P_{e \mu}^{+}$(the upper row) and $P_{e \mu}^{-}$(the lower row) in the $(\Delta \theta, \delta)$ plane, at $L=732,3000$ and $7332 \mathrm{Km}$ for different values of 

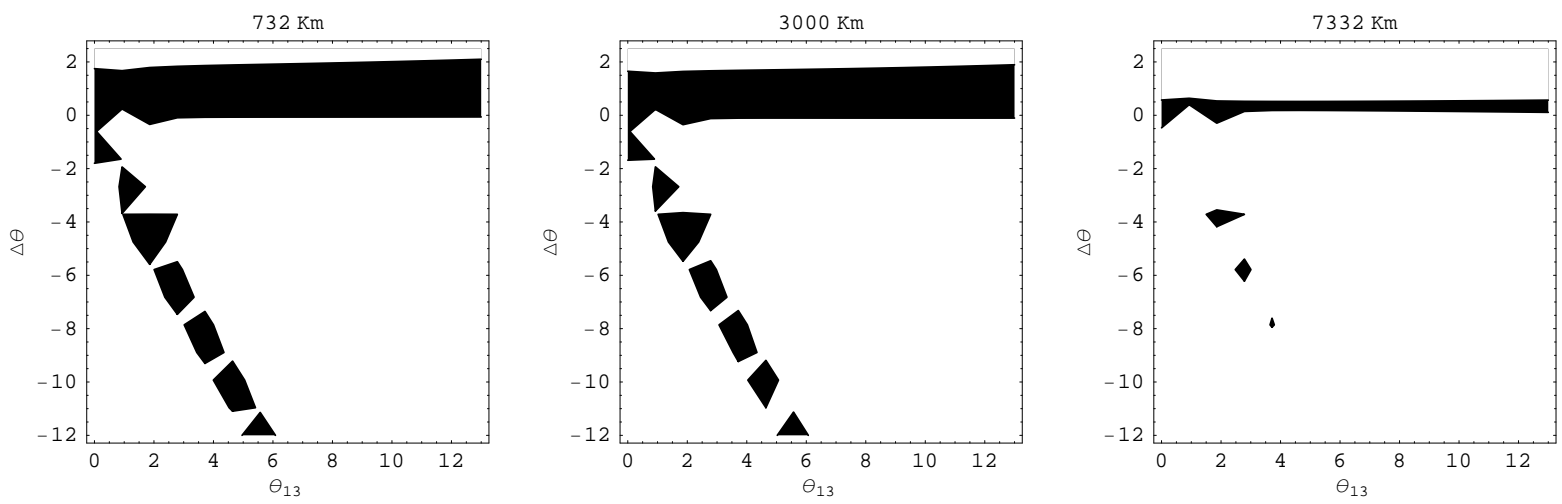

Figure 1: Allowed region in $\Delta \theta$ as a function of $\theta_{13}$ for $E_{\nu}=38$ GeV at three different distances: $L=732,3000$ and $7332 \mathrm{Km}$.

the neutrino energy in the range $E_{\nu} \in[5,50] \mathrm{GeV}$. The input values are $\bar{\theta}_{13}=5^{\circ}$ and $\bar{\delta}=60^{\circ}$. In the upper row (neutrinos), it can be seen that all the equiprobability curves intersect in $\Delta \theta=0^{\circ}, \delta=60^{\circ}$ (namely, $\theta_{13}=\bar{\theta}_{13}$ and $\delta=\bar{\delta}$ ). However, notice that the equiprobability curve for a given neutrino energy intersects the curves corresponding to a different neutrino energy in a second point, at positive $\Delta \theta$ and negative $\delta$. This second intersection depends on the energies of the two curves. In the upper branch of the neutrino equiprobability curves, no second intersection is observed, for these particular values of the input parameters. The results of Fig. 2 may be understood with the help of a perturbative expansion of eqs. (5) and (6) in terms of powers of $\Delta \theta$ (always possible in the allowed region, for $\bar{\theta}_{13}$ large enough). Details on this expansion can be found in App. A.

We can draw some conclusion from what observed in Fig. 2 and from the previous considerations on the energy dependence of the equiprobability curves. In particular, it is to be expected that by fitting experimental data for neutrinos only it should be quite difficult to determine the physical parameters $\left(\bar{\theta}_{13}, \bar{\delta}\right)$ with good accuracy. We expect, instead, that the fitting procedure will identify a low $\chi^{2}$ region whenever the family of equiprobability curves are not well separated, within the experimental energy resolution. In particular, at short distance $(L=732 \mathrm{Km})$ it is to be expected a good determination of $\bar{\theta}_{13}$ (notice that $\Delta \theta$ is generally less than $2^{\circ}$ ) and no determination whatsoever of the CP-violating phase $\bar{\delta}$. At the intermediate distance, $L=3000 \mathrm{Km}$, the equiprobability curves for neutrinos (for this particular set of input parameters) do not depend strongly on the energy in the upper branch, whereas a larger separation can be seen in the lower branch. Therefore, we expect a low $\chi^{2}$ region alongside the upper branch of the equiprobability curves spanning from around the single point corresponding to the physical parameters (at $\Delta \theta=0, \delta=\bar{\delta}$ ) to the (diluted) region where the curves show the second intersection (that by periodicity in the $\delta$ axis happens to be in the lower branch). 

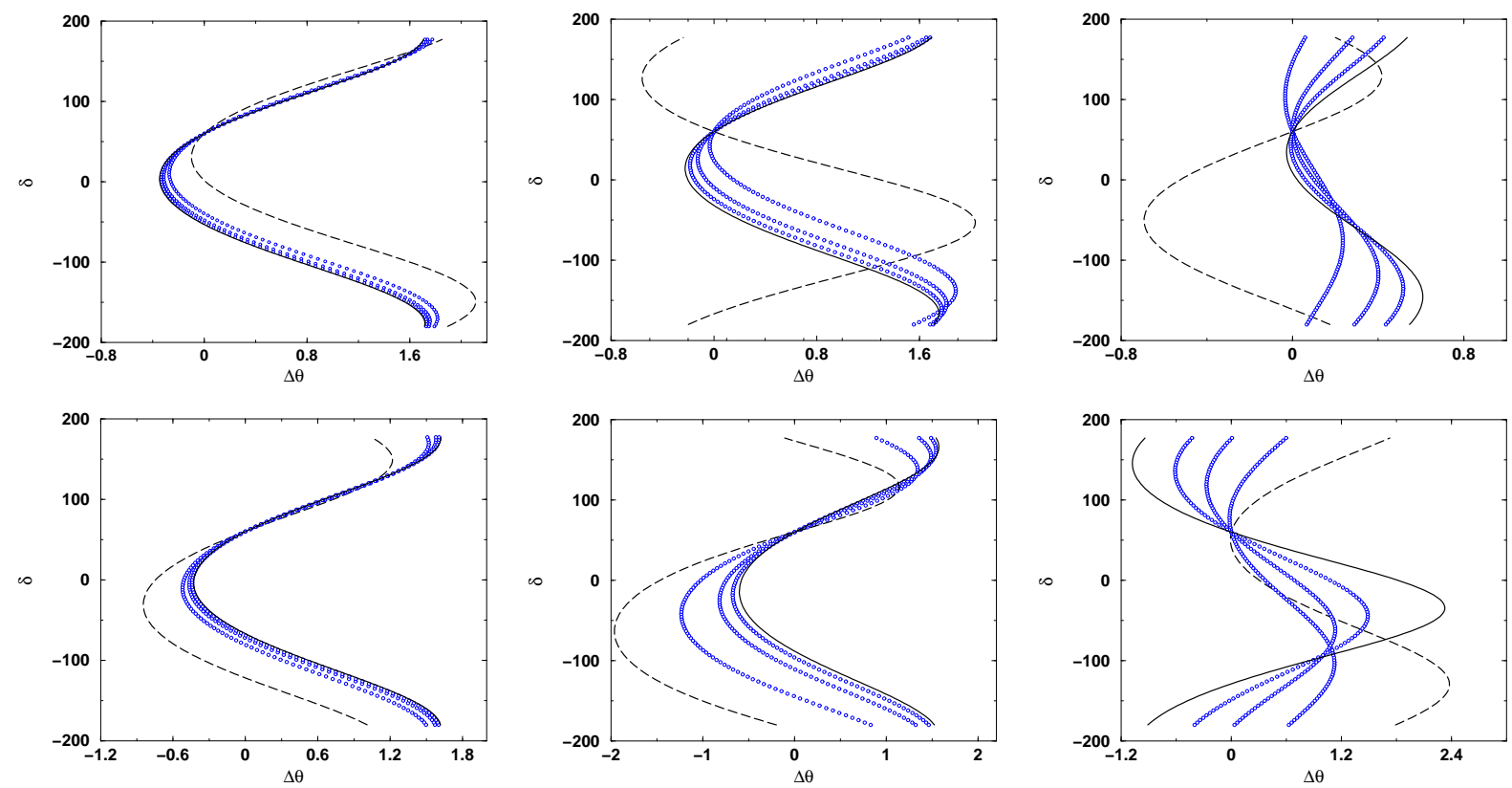

Figure 2: Equiprobability curves in the $(\Delta \theta, \delta)$ plane, for $\bar{\theta}_{13}=5^{\circ}, \bar{\delta}=60^{\circ}, E_{\nu} \in[5,50]$ GeV and $L=732,3000$ and $7332 \mathrm{Km}$. The upper row represents equiprobability curves for neutrinos, the lower row for antineutrinos. The dashed line is $E_{\nu}=5 \mathrm{GeV}$, the solid line is $E_{\nu}=45 \mathrm{GeV}$; the dotted lines lie in between these two.

Finally, at large distance $(L=7332 \mathrm{Km})$ we expect a low $\chi^{2}$ region in the region $\Delta \theta \leq 0.5^{\circ}$ and $\delta \simeq \bar{\delta} \pm 100^{\circ}$ (notice that the small spread in the variable $\theta$ for this baseline is in agreement with Fig. 目).

A great improvement in the reconstruction of the physical parameters from the experimental data is achievable using at the same time neutrino and antineutrino data. This can be seen in Fig. 3, where the equiprobability curves for neutrinos and antineutrinos (for the same input parameters as in Fig. 2) have been superimposed. At short distance, the two family of equiprobability curves overlap for any value of $\delta$, and no improvement is to be expected. However, at the intermediate distance the equiprobability curves for neutrinos and antineutrinos overlap only in the vicinity of the physical point $\Delta \theta=0, \delta=\bar{\delta}$ and in the region of the second intersection, whereas in the intermediate region they are quite well separated, both in the upper and lower branch. We expect, in this case, that the fitting procedure of the whole set of neutrino and antineutrino data will identify two separate low $\chi^{2}$ region, around the physical point and around the region where all the curves show the second intersection. This second allowed region in the parameter space was first observed in 63 and subsequently confirmed in [46, 50, 51]. Finally, at large distance we expect no significant improvement with respect to the previous case.

Notice that these considerations can be drawn by looking at the equiprobability 

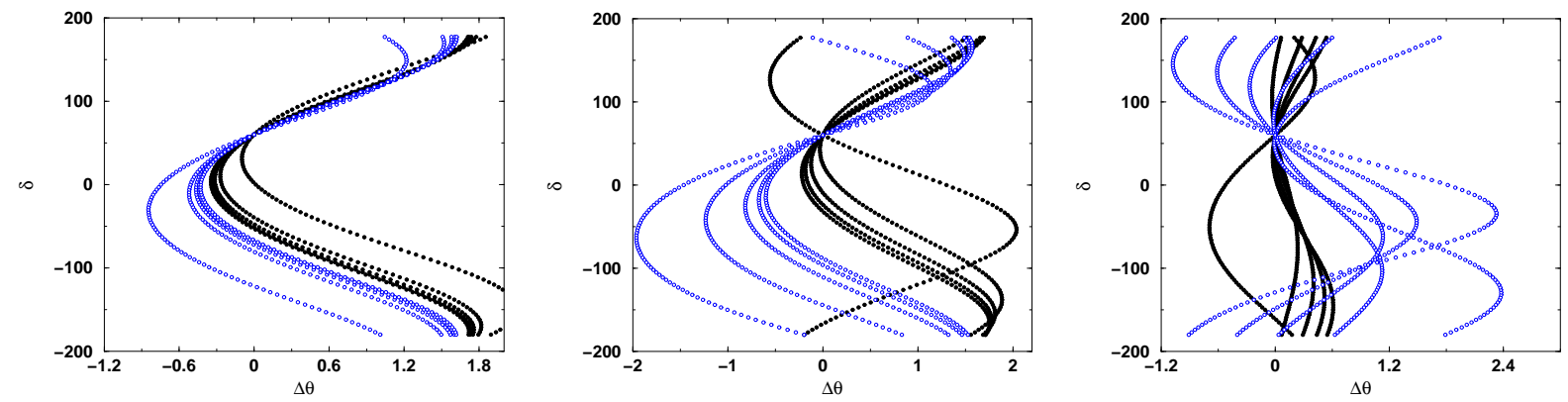

Figure 3: Same as in Fig. \&, but with neutrinos and antineutrinos equiprobability curves superimposed.

curves for neutrinos and antineutrinos, only. We will see in the following section how the theoretical expectation is indeed reproduced in the "experimental data".

\section{Number of "wrong-sign" muons in the detector}

The experimental information is not the transition probability $P_{e \mu}^{ \pm}$but the number of muons with charge opposite to that of the muons circulating in the storage ring, that in the following will be often called "golden" muons. The events are then grouped in bins of energy, with the size of the energy bin depending on the energy resolution $\Delta E$ of the considered detector. In general,

$$
N_{\mu^{\mp}}^{i}\left(\bar{\theta}_{13}, \bar{\delta}\right)=\int_{E_{i}}^{E_{i}+\Delta E} d E \sigma_{\nu_{\mu}\left(\bar{\nu}_{\mu}\right)}(E) P_{e \mu}^{ \pm}\left(E, \bar{\theta}_{13}, \bar{\delta}\right) \frac{d \Phi_{\nu_{e}\left(\bar{\nu}_{e}\right)}(E)}{d E}
$$

is the number of wrong-sign muons in the i-th energy bin for the input pair $\left(\bar{\theta}_{13}, \bar{\delta}\right) ; E$ is the neutrino (antineutrino) energy?. The charged current neutrino and antineutrino interaction rates can be computed using the approximate expressions for the neutrinonucleon cross sections on an isoscalar target,

$$
\sigma_{\nu N} \sim 0.67 \times 10^{-42} \times \frac{E_{\nu}}{G e V} \times m^{2} ; \quad \sigma_{\bar{\nu} N} \sim 0.34 \times 10^{-42} \times \frac{E_{\nu}}{G e V} \times m^{2} .
$$

In the laboratory frame the neutrino fluxes, boosted along the muon momentum vector, are given by:

$$
\begin{aligned}
\frac{d^{2} \Phi_{\nu_{e}, \bar{\nu}_{e}}}{d y d \Omega}= & \frac{24 n_{\mu}}{\pi L^{2} m_{\mu}^{6}} \bar{E}_{\mu}^{4} y^{2}(1-\beta \cos \varphi)\left\{\left[m_{\mu}^{2}-2 \bar{E}_{\mu}^{2} y(1-\beta \cos \varphi)\right]\right. \\
& \left.\mp \mathcal{P}_{\mu}\left[m_{\mu}^{2}-2 \bar{E}_{\mu}^{2} y(1-\beta \cos \varphi)\right]\right\} .
\end{aligned}
$$

\footnotetext{
${ }^{5}$ The neutrino energy can be reconstructed if the considered detector has a hadronic calorimeter capable to measure the energy of the hadronic shower $\left(E_{h a d r}\right)$ in the $\nu N$ CC interactions with good precision.
} 
Here, $\beta=\sqrt{1-\left(m_{\mu} / \bar{E}_{\mu}\right)^{2}}, \bar{E}_{\mu}$ is the parent muon energy, $y=E_{\nu} / \bar{E}_{\mu}, n_{\mu}$ is the number of useful muons per year obtained from the storage ring and $\varphi$ is the angle between the beam axis and the direction pointing towards the detector. In what follows, the fluxes have been integrated in the forward direction with an angular divergence (taken to be constant) $\delta \varphi \sim 0.1 \mathrm{mr}$. The effects of the beam divergence and the QED one-loop radiative corrections to the neutrino fluxes have been properly taken into account in [71]. The overall correction to the neutrino flux has been shown to be of $\mathcal{O}(0.1 \%)$.

In the same approximations as for eq. (1), we get for the number of events per bin:

$$
\left\{\begin{array}{l}
N_{\mu^{-}}^{i}=I_{X_{+}}^{i} \sin ^{2}\left(2 \theta_{13}\right)+\left[I_{Y_{+}^{c}}^{i} \cos \delta+I_{Y_{+}^{s}}^{i} \sin \delta\right] \cos \theta_{13} \sin \left(2 \theta_{13}\right)+I_{Z}^{i}, \\
N_{\mu^{+}}^{i}=I_{X_{-}}^{i} \sin ^{2}\left(2 \theta_{13}\right)+\left[I_{Y_{-}^{c}}^{i} \cos \delta-I_{Y_{-}^{s}}^{i} \sin \delta\right] \cos \theta_{13} \sin \left(2 \theta_{13}\right)+I_{Z}^{i},
\end{array}\right.
$$

where we introduced a short-form notation for the following integrals:

$$
\left\{\begin{aligned}
I_{X_{ \pm}}^{i} & =\int_{E_{i}}^{E_{i}+\Delta E} d E \sigma_{\nu_{\mu}\left(\bar{\nu}_{\mu}\right)} \frac{d \Phi_{\nu_{e}\left(\bar{\nu}_{e}\right)}(E)}{d E} X_{ \pm}(E) \\
I_{Y_{ \pm}^{c}}^{i} & =\int_{E_{i}}^{E_{i}+\Delta E} d E \sigma_{\nu_{\mu}\left(\bar{\nu}_{\mu}\right)} \frac{d \Phi_{\nu_{e}\left(\bar{\nu}_{e}\right)}(E)}{d E} Y_{ \pm}(E) \cos \left(\frac{\Delta_{a t m} L}{2}\right) \\
I_{Y_{ \pm}^{s}}^{i} & =\int_{E_{i}}^{E_{i}+\Delta E} d E \sigma_{\nu_{\mu}\left(\bar{\nu}_{\mu}\right)} \frac{d \Phi_{\nu_{e}\left(\bar{\nu}_{e}\right)}(E)}{d E} Y_{ \pm}(E) \sin \left(\frac{\Delta_{a t m} L}{2}\right) \\
I_{Z}^{i} & =\int_{E_{i}}^{E_{i}+\Delta E} d E \sigma_{\nu_{\mu}\left(\bar{\nu}_{\mu}\right)} \frac{d \Phi_{\nu_{e}\left(\bar{\nu}_{e}\right)}(E)}{d E} Z(E)
\end{aligned}\right.
$$

For a fixed energy bin and fixed input parameters $\left(\bar{\theta}_{13}, \bar{\delta}\right)$, we can draw a continuous curve of equal number of events in the $(\Delta \theta, \delta)$ plane,

$$
N_{\mu^{ \pm}}^{i}\left(\theta_{13}, \delta\right)=N_{\mu^{ \pm}}^{i}\left(\bar{\theta}_{13}, \bar{\delta}\right)
$$

as it was the case for the transition probability, eq. (3).

We therefore get an implicit equation in $\delta$,

$$
F(\delta)=G\left(\theta_{13}, \bar{\theta}_{13}, \bar{\delta}\right)
$$

where

$$
\begin{cases}F(\delta) & =\cos \delta \pm\left(\frac{I_{Y_{ \pm}^{s}}^{i}}{I_{Y_{ \pm}^{c}}^{i}}\right) \sin \delta \\ G\left(\theta_{13}, \bar{\theta}_{13}, \bar{\delta}\right) & =\left(\frac{I_{X_{ \pm}}^{i}}{I_{Y_{ \pm}^{c}}^{i}}\right) f\left(\theta_{13}, \bar{\theta}_{13}\right)+F(\bar{\delta}) g\left(\theta_{13}, \bar{\theta}_{13}\right)\end{cases}
$$

and $f\left(\theta_{13}, \bar{\theta}_{13}\right)$ and $g\left(\theta_{13}, \bar{\theta}_{13}\right)$ are the $\theta$-dependent functions introduced in eq. (5). Solving for $\delta$,

$$
\delta=F^{-1}\left[G\left(\theta_{13}, \bar{\theta}_{13}, \bar{\delta}\right)\right]
$$

we get equal-number-of-events curves $(\mathrm{ENE})$ in the $(\Delta \theta, \delta)$ plane, see Fig. 1 . 

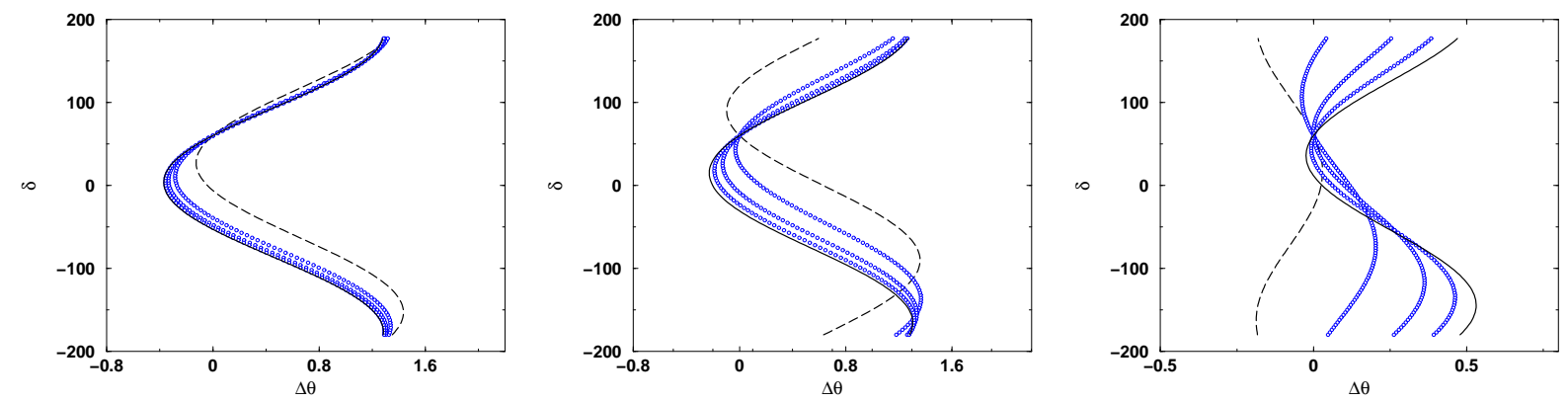

Figure 4: Equal-number-of-events (ENE) curves in the $(\Delta \theta, \delta)$ plane for neutrinos, for $\bar{\theta}_{13}=5^{\circ}, \bar{\delta}=60^{\circ}, E_{\nu} \in[5,50]$ GeV and $L=732,3000$ and $7332 \mathrm{Km}$. The dashed line represents the first energy bin, $E_{\nu} \in[0,10] \mathrm{GeV}$, the solid line the last energy bin, $E_{\nu} \in[40,50] \mathrm{GeV}$; the dotted lines lie in between these two.

The problem arises in the reconstruction of the physical parameters from a data set consisting of some given number of events per bin, for a given number of bins (depending on the specific detector energy resolution). As it was the case for the equiprobability curves in the previous section, all the ENE curves intersect in the physical point $(\Delta \theta=0, \delta=\bar{\delta})$ and any given couple of curves intersect in a second point in the same region as in Fig. 2. As it can be seen in Fig. 14, the second intersection differs when considering different couples of curves, but lies always in a restricted area of the $(\Delta \theta, \delta)$ plane, the specific location of this region depending on the input parameters $\left(\bar{\theta}_{13}, \bar{\delta}\right)$, see eqs. (25) and (26). The $\chi^{2}$ analysis of the data will therefore identify two allowed regions: the "physical" one (around the physical value, $\bar{\theta}_{13}, \bar{\delta}$ ) and the "clone" solution, spanning all the area where a second intersection between any two ENE curves occurs. This is the source of the ambiguity pointed out in [63].

In the remaining of this section, we apply the analysis in energy bins of 40, 63. Let $N_{i, p}^{\lambda}$ be the total number of wrong-sign muons detected when the factory is run in polarity $p=\mu^{+}, \mu^{-}$, grouped in energy bins specified by the index $i$, and three possible distances, $\lambda=1,2,3$ (corresponding to $L=732 \mathrm{Km}, L=3000 \mathrm{Km}$ and $L=7332 \mathrm{Km}$, respectively). In order to simulate a typical experimental situation we generate a set of "data" $n_{i, p}^{\lambda}$ as follows: for a given value of the oscillation parameters, the expected number of events, $N_{i, p}^{\lambda}$, is computed; taking into account backgrounds and detection efficiencies per bin, $b_{i, p}^{\lambda}$ and $\epsilon_{i, p}^{\lambda}$, we then perform a gaussian (or poissonian for $N_{i, p}^{\lambda} \leq 10$ events) smearing to mimic the statistical uncertainty:

$$
n_{i, p}^{\lambda}=\operatorname{Smear}\left(N_{i, p}^{\lambda} \epsilon_{i, p}^{\lambda}+b_{i, p}^{\lambda}\right) .
$$

Finally, "data" are fitted to the theoretical expectation as a function of the neutrino parameters under study, using a gaussian $\chi^{2}$ minimization:

$$
\chi_{\lambda}^{2}=\sum_{p} \sum_{i}\left(\frac{n_{i, p}^{\lambda}-N_{i, p}^{\lambda}}{\delta n_{i, p}^{\lambda}}\right)^{2},
$$


where $\delta n_{i, p}^{\lambda}$ is the statistical error for $n_{i, p}^{\lambda}$ (errors on background and efficiencies are neglected) or a poissonian $\chi^{2}$ minimization:

$$
\chi_{\lambda}^{2}=-2 \sum_{p} \sum_{i}\left[\left(n_{i, p}^{\lambda}-N_{i, p}^{\lambda}\right)-n_{i, p}^{\lambda} \log \left(\frac{n_{i, p}^{\lambda}}{N_{i, p}^{\lambda}}\right)\right]
$$

whenever events are Poisson-distributed around the theoretical values (see 60 and refs. therein). We verified that the fitting of theoretical numbers to the smeared ("experimental") ones is able to reproduce the values of the input parameters (the best fit always lies within a restricted region around $\bar{\theta}_{13}, \bar{\delta}$ ).

The following "reference set-up" has been considered: neutrino beams resulting from the decay of $2 \times 10^{20} \mu^{+}$'s and $\mu^{-}$'s per year in a straight section of an $\bar{E}_{\mu}=50$ $\mathrm{GeV}$ muon accumulator. An experiment with a realistic 40 Kton detector of magnetized iron and five years of data taking for each polarity is envisaged. Detailed estimates of the corresponding expected backgrounds and efficiencies have been included in the analysis, following [66. Notice that this set-up is exactly the same of 40, 63.

In the first row of Figs. 56, we present the results of the fit to five bins of data for decaying muons of one single polarity, $\mu^{-}$. The energy resolution of the detector is $\Delta E_{\nu}=10 \mathrm{GeV}$. In all cases we observe the pattern depicted in the previous section, with a good determination of $\bar{\theta}_{13}$ and an extremely poor determination of $\bar{\delta}$. In the second row we fit to five bins of data for decaying muons of both polarities. The results follow again the theoretical analysis of this and of the previous section and are in perfect agreement with what presented in [63]. In particular, notice how at the intermediate distance it is possible now to reconstruct $\delta$ with an error of tens of degrees in two separate regions of the parameter space. 

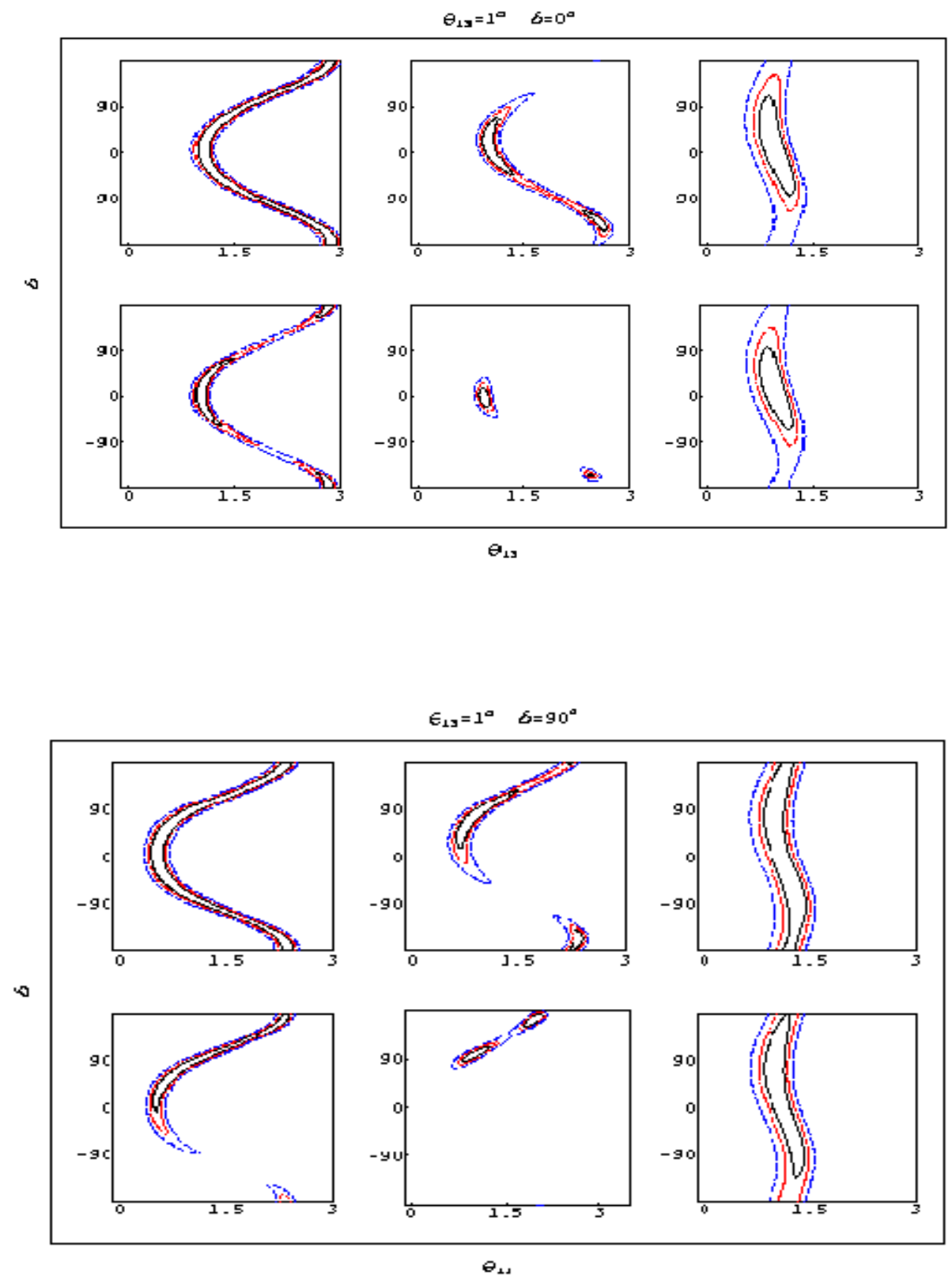

Figure 5: 68.5, 90 and $99 \%$ C.L. contours resulting from a $\chi^{2}$ fit of $\theta_{13}$ and $\delta$, for $\bar{\theta}_{13}=1^{\circ}$ and $\bar{\delta}=0^{\circ}$ (up) and $\bar{\delta}=90^{\circ}$ (down). For each considered input parameters couple, the two rows represent: from left to right, $L=732,3000$ and $7332 \mathrm{Km}$; the upper row is $N_{\mu^{+}}^{i}, i=1, \ldots, 5$; the lower row is $N_{\mu^{+}}^{i}$ and $N_{\mu^{-}}^{i}, i=1, \ldots, 5$. 

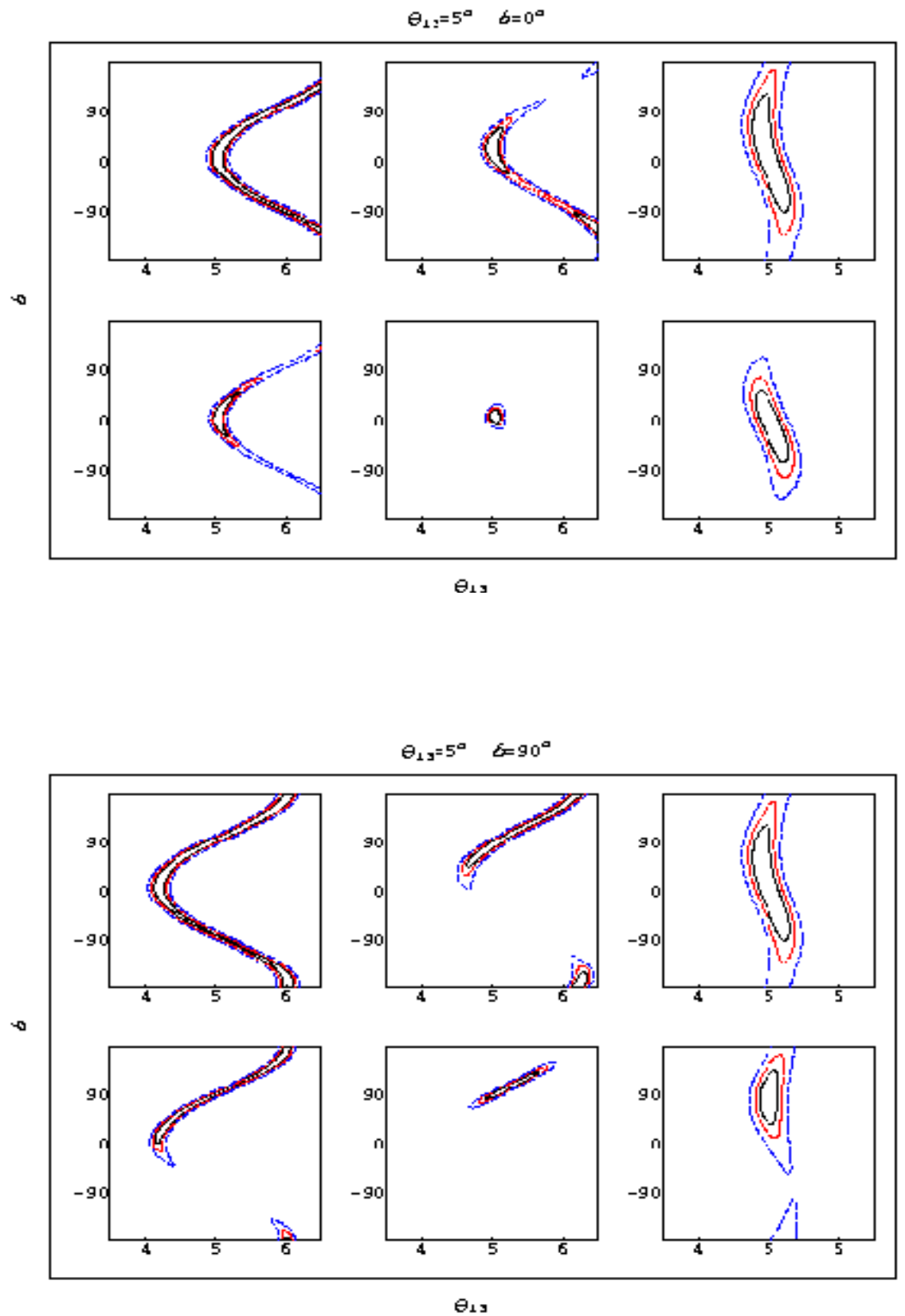

Figure 6: 68.5, 90 and $99 \%$ C.L. contours resulting from a $\chi^{2}$ fit of $\theta_{13}$ and $\delta$, for $\bar{\theta}_{13}=5^{\circ}$ and $\bar{\delta}=0^{\circ}$ (up) and $\bar{\delta}=90^{\circ}$ (down). For each considered input parameters couple, the two rows represent: from left to right, $L=732,3000$ and $7332 \mathrm{Km}$; the upper row is $N_{\mu^{+}}^{i}, i=1, \ldots, 5$; the lower row is $N_{\mu^{+}}^{i}$ and $N_{\mu^{-}}^{i}, i=1, \ldots, 5$. 


\section{The $\nu_{e} \rightarrow \nu_{\tau}$ equiprobability and ENE curves}

We present in this section the possibility to use a different channel, namely the $\nu_{e} \rightarrow \nu_{\tau}$ oscillation probability, to improve the reconstruction of the physical parameters $\left(\bar{\theta}_{13}, \bar{\delta}\right)$ in combination with the results for the $\nu_{e} \rightarrow \nu_{\mu}$ transition described in [40, 63] and in the previous section.

The $\nu_{e} \rightarrow \nu_{\tau}$ oscillation probability at second order in perturbation theory in $\theta_{13}$, $\Delta_{\odot} / \Delta_{a t m}, \Delta_{\odot} / A$ and $\Delta_{\odot} L$ is:

$$
P_{e \tau}^{ \pm}\left(\bar{\theta}_{13}, \bar{\delta}\right)=X_{ \pm}^{\tau} \sin ^{2}\left(2 \bar{\theta}_{13}\right)-Y_{ \pm} \cos \left(\bar{\theta}_{13}\right) \sin \left(2 \bar{\theta}_{13}\right) \cos \left( \pm \bar{\delta}-\frac{\Delta_{a t m} L}{2}\right)+Z^{\tau}
$$

where \pm refers to neutrinos and antineutrinos, respectively, and

$$
\left\{\begin{array}{l}
X_{ \pm}^{\tau}=\cos ^{2}\left(\theta_{23}\right)\left(\frac{\Delta_{a t m}}{B_{\mp}}\right)^{2} \sin ^{2}\left(\frac{B_{\mp} L}{2}\right) \\
Y_{ \pm}=\sin \left(2 \theta_{12}\right) \sin \left(2 \theta_{23}\right)\left(\frac{\Delta_{\odot}}{A}\right)\left(\frac{\Delta_{a t m}}{B_{\mp}}\right) \sin \left(\frac{A L}{2}\right) \sin \left(\frac{B_{\mp} L}{2}\right) \\
Z^{\tau}=\sin ^{2}\left(\theta_{23}\right) \sin ^{2}\left(2 \theta_{12}\right)\left(\frac{\Delta_{\odot}}{A}\right)^{2} \sin ^{2}\left(\frac{A L}{2}\right)
\end{array}\right.
$$

with $Z^{\tau}=Z_{+}^{\tau}=Z_{-}^{\tau}$. Notice that $X_{ \pm}^{\tau}$ and $Z^{\tau}$ differs from the corresponding coefficients for the $\nu_{e} \rightarrow \nu_{\mu}$ transition for the $\cos \theta_{23} \leftrightarrow \sin \theta_{23}$ exchange, only. The $Y_{ \pm}$term is identical for the two channels, but it appears with an opposite sign. This sign difference in the $Y$-term is crucial, as it determines a different shape in the $(\Delta \theta, \delta)$ plane for the two sets of equiprobability curves.
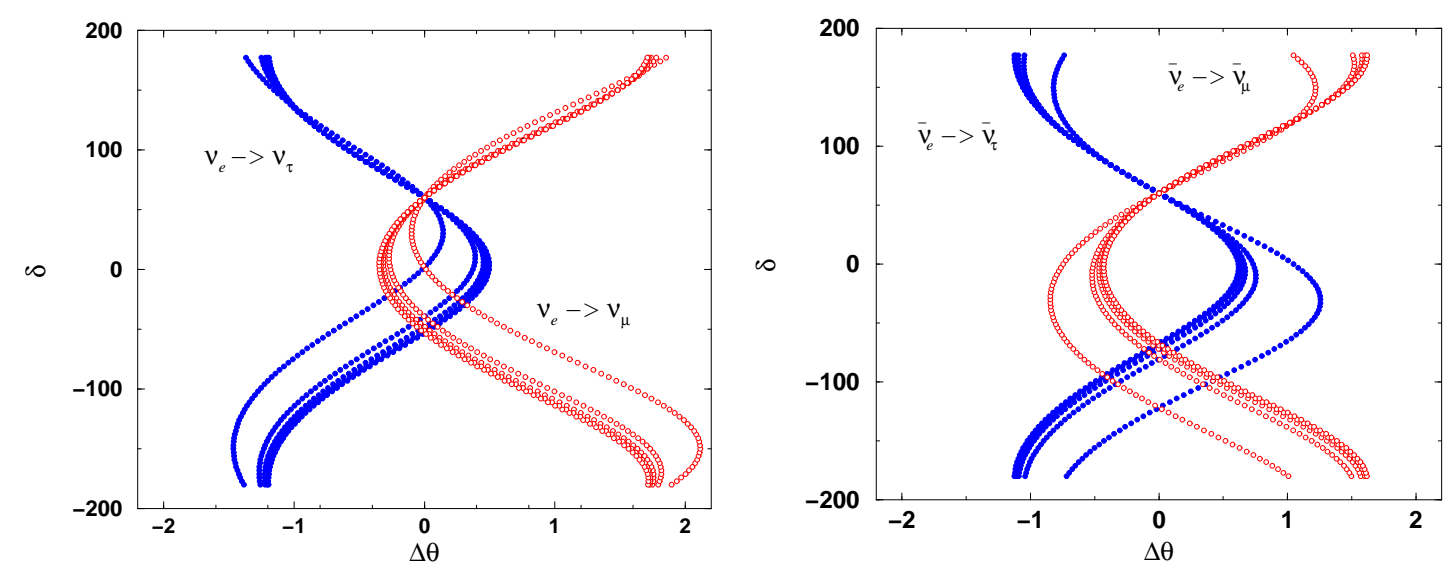

Figure 7: Equiprobability curves in the $(\Delta \theta, \delta)$ plane, for $\bar{\theta}_{13}=5^{\circ}, \bar{\delta}=60^{\circ}, E_{\nu} \in[5,50]$ GeV and $L=732 \mathrm{Km}$ for the $\nu_{e} \rightarrow \nu_{\mu}$ and $\nu_{e} \rightarrow \nu_{\tau}$ oscillation (neutrinos on the left, antineutrinos on the right). 
In Fig. 7, we superimposed the equiprobability curves for the $\nu_{e} \rightarrow \nu_{\tau}$ and $\nu_{e} \rightarrow \nu_{\mu}$ oscillations at a fixed distance, $L=732 \mathrm{Km}$, with input parameters $\bar{\theta}_{13}=5^{\circ}$ and $\bar{\delta}=60^{\circ}$, for different values of the energy, $E_{\nu} \in[5,50] \mathrm{GeV}$. The effect of the different sign in front of the $Y$-term in eqs. (回) and (20) can be seen in the opposite shape in the $\left(\theta_{13}, \delta\right)$ plane of the $\nu_{e} \rightarrow \nu_{\tau}$ curves with respect to the $\nu_{e} \rightarrow \nu_{\mu}$ ones. Notice that all the curves of both families met in the "physical" point, $\theta_{13}=\bar{\theta}_{13}, \delta=\bar{\delta}$, and that now three would-be "clone" regions (i.e., the spread regions where the intersections of any given couple of equiprobability curves lie) can be seen.

As a final comment we signal that, if $\theta_{13}$ is not extremely small (in such a way that the $X_{ \pm}$and $X_{ \pm}^{\tau}$ terms dominate over the $Y_{ \pm}$terms in eqs. (11) and (20)), the combined measurement of $\nu_{e} \rightarrow \nu_{\mu}$ and $\nu_{e} \rightarrow \nu_{\tau}$ transitions could in principle solve the $\left[\theta_{23}, \pi / 2-\theta_{23}\right]$ ambiguity.

To follow the line of reasoning adopted for the $\nu_{e} \rightarrow \nu_{\mu}$ channel, we should now discuss how the $\nu_{e} \rightarrow \nu_{\tau}$ channel can be used in a realistic experiment. A number of modifications with respect to the case of the "golden" channel should be taken into account.

First, the approximate expressions for the neutrino-nucleon cross section on an isoscalar target, eq. (9), are no longer appropriate in the case of a $\nu_{\tau} \mathrm{CC}$ interaction inside the detector. In this case we used the reported values for the $\nu_{\tau} N$ cross-section [72 that have been applied in the CHORUS and OPERA experiment to compute the expected number of CC $\tau$ events. The considered cross-section includes $\tau$ mass effects in the DIS region following [73] (see App. B for details), as well as the elastic and quasi-elastic contributions to the cross section.

Second, the $\tau$ will decay in flight into a muon of the same charge and two neutrinos, with a branching ratio $B R(\tau \rightarrow \mu) \simeq 0.17$, [19]. This "silver" wrong-sign muon is the experimental signal we are looking for, to be identified and to be separated from the "golden" wrong-sign muons originated from $\nu_{\mu} \mathrm{CC}$ interactions 9 . The first tool to distinguish the two sets of wrong-sign muons is their different energy distribution (see App. $\mathbb{B}$ for details on the differential decay rate). It has been shown in that in the magnetized iron detector considered in the previous section, muons from $\tau$ decay cannot be distinguished from the main background represented by muons from charmed mesons decay by means of kinematical cuts. In order to take advantage of this channel, we should therefore use a different kind of detector: for this reason we concentrate in the remaining of the paper on a lead-emulsion detector, where the observation of the $\tau$ decay vertex allows to distinguish "golden" and "silver" wrong-sign muons, and the latter from the charmed mesons decay background. We must mention that the $\nu_{e} \rightarrow \nu_{\tau}$ oscillations were previously considered in [48] for a liquid argon detector, using kinematical cuts to identify $\tau$ 's. It could be of interest to explore further the possibility of using "silver" muon events in such a detector to reduce or eliminate the

\footnotetext{
${ }^{6}$ We adopt the nick-name of "silver" muon events due to the lesser statistical significance with respect to "golden" ones.
} 
$\left(\theta_{13}, \delta\right)$ ambiguity.

In what follows, we consider an OPERA-like detector with a mass of 2 Kton and spectrometers capable of muon charge identification (see the OPERA proposal for details, [74]) located at $L=732 \mathrm{Km}$ down the neutrino source (obviously, in the back of our mind we are thinking of the CNGS set-up). The results for both the "golden" and the "silver" channel at the near emulsion detector will be combined with results for the "golden" channel obtained with the magnetized iron detector located at the optimal distance for the measurement of leptonic CP violation, $L=3000 \mathrm{Km}$.

In this paper, we will first restrict ourselves to an ideal OPERA-like detector with perfect efficiency and no background. Afterwards, we take into account the realistic estimates of the energy-dependent reconstruction efficiency and of the most relevant backgrounds[? Eventually, we will consider how an increase in the detector mass or an improvement on the signal/noise ratio affects our results.

Schematically, starting from a positive charged muon in the storage ring, "silver" muons are obtained by the following chain:

$$
\mu^{+} \rightarrow\left\{\begin{array}{l}
e^{+} \\
\bar{\nu}_{\mu} \\
\nu_{e} \rightarrow \nu_{\tau} \rightarrow \tau^{-} \rightarrow \mu^{-}
\end{array}\right.
$$

whereas "golden" muons come from:

$$
\mu^{+} \rightarrow\left\{\begin{array}{l}
e^{+} \\
\bar{\nu}_{\mu} \\
\nu_{e} \rightarrow \nu_{\mu} \rightarrow \mu^{-}
\end{array}\right.
$$

If we group the events in bins of the final muon energy $E_{\mu}$, with the size of the energy bin depending on the energy resolution $\Delta E_{\mu}$ of the considered detector, the number of "golden" muons in the i-th energy bin for the input pair $\left(\bar{\theta}_{13}, \bar{\delta}\right)$ and for a parent muon energy $\bar{E}_{\mu}$ is:

$$
N_{\mu^{\mp}}^{g}\left(\bar{\theta}_{13}, \bar{\delta}\right)=\left\{\frac{d \sigma_{\nu_{\mu}\left(\bar{\nu}_{\mu}\right)}\left(E_{\mu}, E_{\nu}\right)}{d E_{\mu}} \otimes P_{e \mu}^{ \pm}\left(E_{\nu}, \bar{\theta}_{13}, \bar{\delta}\right) \otimes \frac{d \Phi_{\nu_{e}\left(\bar{\nu}_{e}\right)}\left(E_{\nu}, \bar{E}_{\mu}\right)}{d E_{\nu}}\right\}_{E_{i}}^{E_{i}+\Delta E_{\mu}}
$$

(remember that $P^{ \pm}$is the oscillation probability for neutrinos and antineutrinos, respectively, see Sect. 21), whereas the number of "silver" muons in the i-th energy bin

\footnotetext{
${ }^{7}$ A dedicated careful analysis of the "silver" muons reconstruction efficiency and of the background as a function of the neutrino energy for this specific detector is currently under progress, [75]. A key issue is the maximum affordable amount of charge discrimination, both for the emulsion and the magnetized iron detector. Estimates can be found in [66] for the magnetized iron detector and in Fig. 86 of [74] for the emulsion detector.
} 


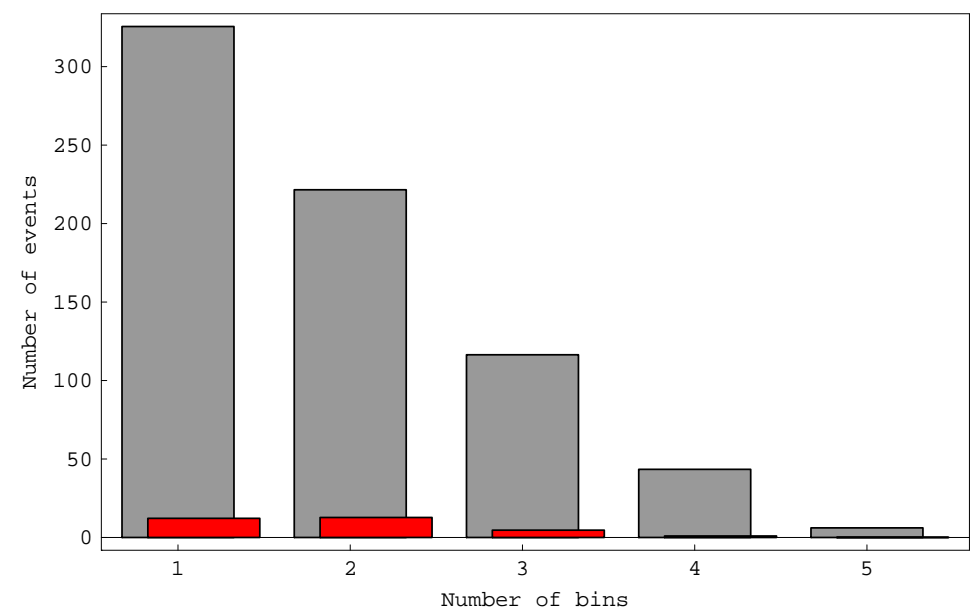

Figure 8: Comparison of the number of "golden" muons (lightest bars) and "silver" muons (darkest bars), for a parent muon energy $\bar{E}_{\mu}=50 \mathrm{GeV}$ and input parameters $\bar{\theta}_{13}=5^{\circ}, \bar{\delta}=60^{\circ}$ and a near detector $(L=732 \mathrm{Km})$ with a mass of 2 Kton and perfect reconstruction efficiency for both channels.

is:

$$
\begin{aligned}
N_{\mu^{\mp}}^{s}\left(\bar{\theta}_{13}, \bar{\delta}\right)=B R(\tau & \rightarrow \mu)\left\{\left[\frac{d N_{\mu^{\mp}}\left(E_{\mu}, E_{\tau}\right)}{d E_{\mu}} \otimes \frac{d \sigma_{\nu_{\tau}\left(\bar{\nu}_{\tau}\right)}\left(E_{\tau}, E_{\nu}\right)}{d E_{\tau}}\right]\right. \\
& \left.\otimes P_{e \tau}^{ \pm}\left(E_{\nu}, \bar{\theta}_{13}, \bar{\delta}\right) \otimes \frac{d \Phi_{\nu_{e}\left(\bar{\nu}_{e}\right)}\left(E_{\nu}, \bar{E}_{\mu}\right)}{d E_{\nu}}\right\}_{E_{i}}^{E_{i}+\Delta E_{\mu}}
\end{aligned}
$$

In both equations, $\otimes$ stands for a convolution integral on the intermediate energy: for example,

$$
B R(\tau \rightarrow \mu)\left[\frac{d N_{\mu^{\mp}}\left(E_{\mu}, E_{\tau}\right)}{d E_{\mu}} \otimes \frac{d \sigma_{\nu_{\tau}\left(\bar{\nu}_{\tau}\right)}\left(E_{\tau}, E_{\nu}\right)}{d E_{\tau}}\right]
$$

gives the number of muons in the $\mathrm{i}$-th bin in the final muon energy $E_{\mu}$, for a given neutrino energy $E_{\nu}$ (see App. B for details). In Fig. 8 we present a direct comparison of "golden" and "silver" muons grouped in five energy bins with $\Delta E_{\mu}=10 \mathrm{GeV}$, for a parent muon energy $\bar{E}_{\mu}=50 \mathrm{GeV}$ with input parameters $\bar{\theta}_{13}=5^{\circ}, \bar{\delta}=60^{\circ}$. We consider here a near detector $(L=732 \mathrm{Km})$ with a mass of 2 Kton and perfect reconstruction efficiency for both channels. Notice that in Fig. 8 we have not included quasi-elastic and resonance contributions to the $\nu N$ cross-section that could enhance $\tau$ production at low neutrino energy.

The total number of events for these parameters are $N_{\mu^{-}}^{g} \sim 700$ and $N_{\mu^{-}}^{s} \sim 30$. The strong reduction in the number of "silver" muons with respect to the "golden" muons 
with the same input parameters depends on the suppression due to the $B R(\tau \rightarrow \mu)$ branching ratio and to the different $\nu N$ DIS cross-section for muons and taus.
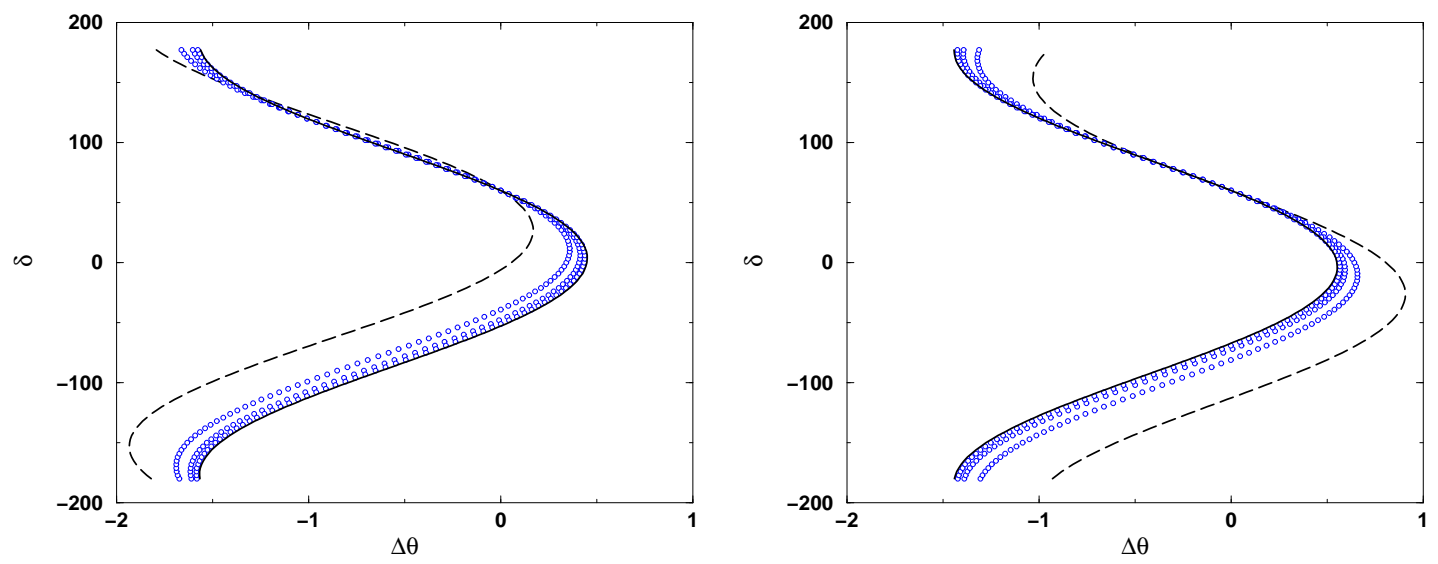

Figure 9: Equal-number-of-event-curves in the $(\Delta \theta, \delta)$ plane, for $E_{\nu} \in[5,50]$ GeV and and $L=732 \mathrm{Km}$ in the case of $\nu_{e} \rightarrow \nu_{\tau}$ oscillation (neutrinos on the left, antineutrinos on the right), for $\bar{\theta}_{13}=5^{\circ}$ and $\bar{\delta}=60^{\circ}$.

Following the same procedure used to get the "golden" muons ENE curves presented in Fig. 团 we can compute ENE curves for "silver" muons. These curves are reported in Fig. 9 in the case of $L=732 \mathrm{Km}$.

In Fig. 10 we superimpose "golden" and "silver" ENE curves for $L=732 \mathrm{Km}$ and $\bar{\theta}_{13}=5^{\circ}, \bar{\delta}=60^{\circ}$. Notice how, as it was expected from the equiprobability curves analysis, the two sets of curves have opposite concavity in the $(\Delta \theta, \delta)$ plane. As for the equiprobability curves, all lines met in the "physical" point. Therefore, a combined $\chi^{2}$ analysis of the two sets of data should present a well defined global minimum around the "physical" region, whereas the local minima situated in the three "clone" regions are considerably raised with respect to what presented in the previous section where only the "golden" muon signal was considered.

\section{Combination of "golden" and "silver" muon events}

We follow the analysis in energy bins outlined in the previous section and in [40, 63]: we produce a theoretical data set $\left(N_{\mu^{ \pm}}^{g}, N_{\mu^{ \pm}}^{s}\right)$ for fixed input parameters $\bar{\theta}_{13}, \bar{\delta}$ and then we smear it as in eq. (17) to obtain an "experimental" data set $\left(n_{\mu^{ \pm}}^{g}, n_{\mu^{ \pm}}^{s}\right)$. Finally, "experimental" data are fitted and 68.5, 90 and $99 \%$ C.L. contours in the $\left(\theta_{13}, \delta\right)$ plane are drawn.

In Fig. 11 we present the results of this analysis comparing two different possibilities: in the upper row we combine two realistic magnetized iron detectors at $L=732$ and 


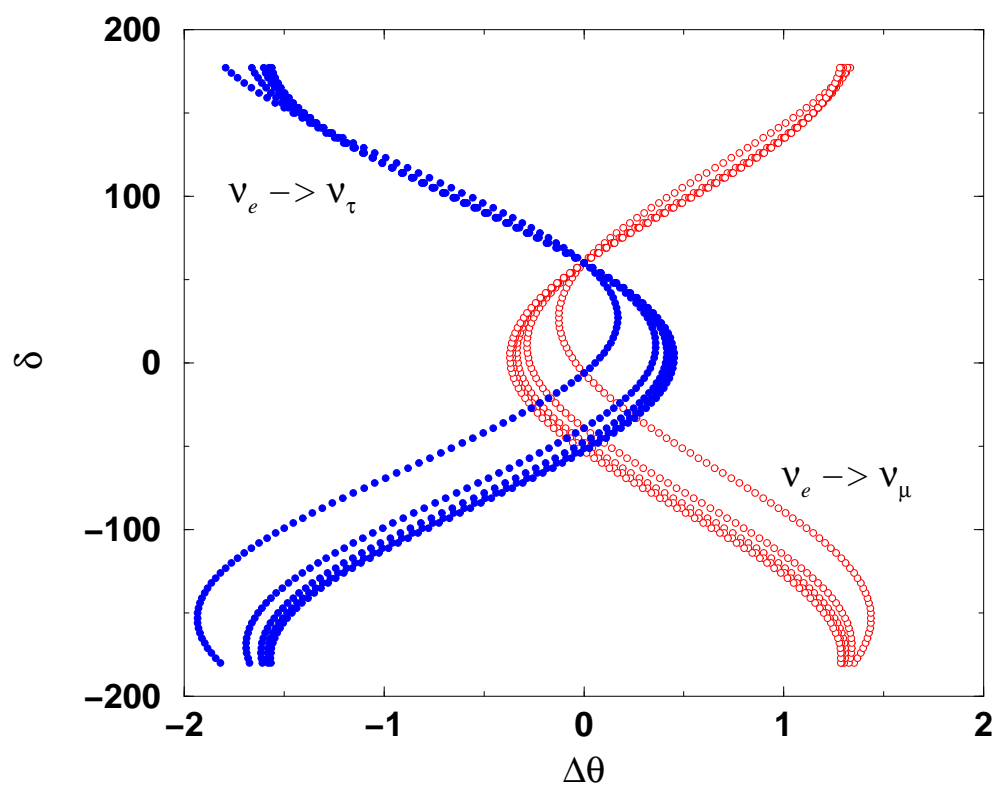

Figure 10: Superposition of the equal-number-of-events curves for the transition $\nu_{e} \rightarrow$ $\nu_{\mu}$ (light lines) and $\nu_{e} \rightarrow \nu_{\tau}$ (dark lines), for $L=732 \mathrm{Km}$ and $\bar{\theta}_{13}=5^{\circ}, \bar{\delta}=60^{\circ}$.

$L=3000 \mathrm{Km}$; in the lower row we combine an ideal OPERA-like detector at $L=732$ $\mathrm{Km}$ and a realistic magnetized iron detector at $L=3000 \mathrm{Km}$.

First, we present our results for the combination of the two iron detectors (where only "golden" muons, $N_{\mu^{ \pm}}^{g}$, can be used). We draw in each figure the contours for different input parameters: three values for $\bar{\theta}_{13}=1^{\circ}, 6^{\circ}$ and $11^{\circ}$ and three values for the phase $\bar{\delta}=-90^{\circ}, 0^{\circ}$ and $90^{\circ}$. In each figure, therefore, fits to nine input parameter

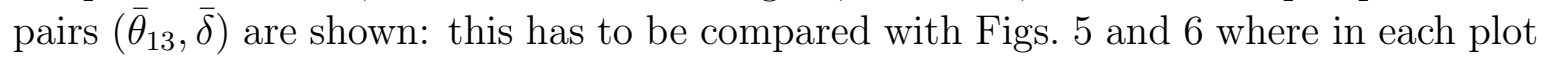
the results of a fit to one single input parameter pair was presented.

On the left, only five years of data taking for $\mu^{+}$circulating in the storage ring are considered. Notice that for any given input pair $\theta_{13}$ is always reconstructed within a $2^{\circ}$ error; on the contrary, roughly any value for the $\mathrm{CP}$-violating phase is allowed. The situation is drastically improved on the right, where five years of data taking for each muon polarization are considered. In this case, the phase $\delta$ is reconstructed with a precision of tens of degrees for all values of the input parameters. Notice, however, how some "clone" region is still present at $90 \%$ C.L. (e.g., for $\bar{\theta}_{13}=1^{\circ}, \bar{\delta}=90^{\circ}$ the small region around $\theta_{13}=2^{\circ}, \delta=150^{\circ}$; for $\bar{\theta}_{13}=1^{\circ}, \bar{\delta}=0^{\circ}$ the small region around $\theta_{13}=2.5^{\circ}, \delta=-150^{\circ}$; see also Fig. 50. These results can be easily understood in terms of the theoretical analysis of the equiprobability and equal-number-of-events curves for the $\nu_{e} \rightarrow \nu_{\mu}$ channel of Sects. 2 and 8 .

We present now results for the combination of a near emulsion detector (with both "golden" and "silver" muons, $N_{\mu^{ \pm}}^{g}$ and $N_{\mu^{ \pm}}^{s}$ ) and a not-so-far iron detector (with 

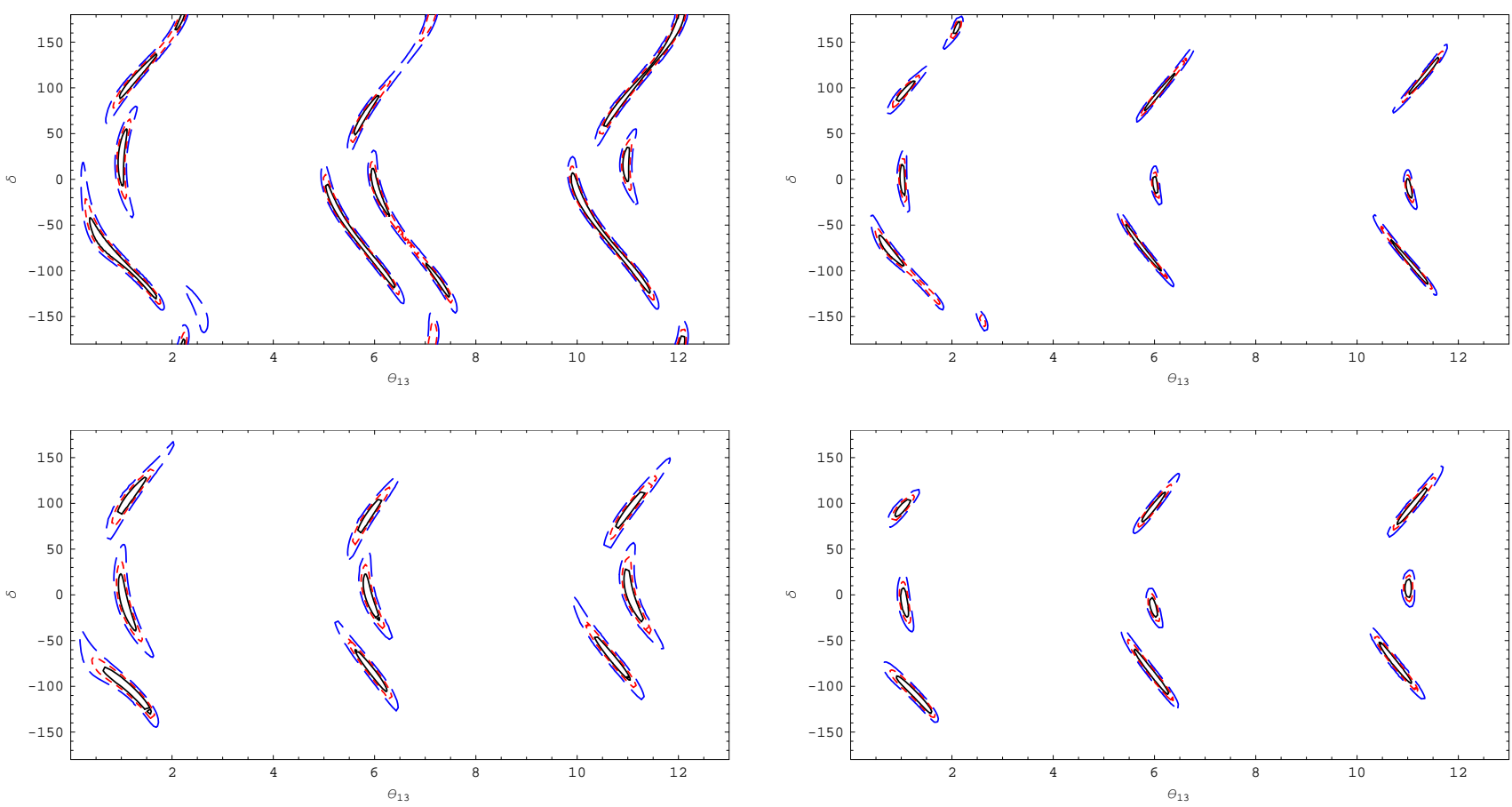

Figure 11: $68.5,90$ and $99 \%$ C.L. contours resulting from a $\chi^{2}$ fit of $\theta_{13}$ and $\delta$, for $\bar{\theta}_{13}=1^{\circ}, 6^{\circ}$ and $11^{\circ}$, and $\bar{\delta}=-90^{\circ}, 0^{\circ}$ and $90^{\circ}$, for the combination of two iron detectors (upper row) or one iron detector and one emulsion detector (lower row). Upper row: iron detector at $L=732$ and $3000 \mathrm{Km}$ : a) $N_{\mu^{+}}^{g}$; b) $N_{\mu^{+}}^{g}$ and $N_{\mu^{-}}^{g}$; Lower row: iron detector at $L=3000 \mathrm{Km}$ and emulsion detector at $L=732 \mathrm{Km}$ : a) $N_{\mu^{+}}^{g}+N_{\mu^{+}}^{s}$; b) $N_{\mu^{+}}^{g}+N_{\mu^{+}}^{s}$ and $N_{\mu^{-}}^{g}$.

"golden" muons, only). On the left, again only five years of data taking for $\mu^{+}$circulating in the storage ring are considered. Notice that a significant reduction in the reconstruction errors on the phase $\delta$ is already achieved. On the right, we simply add to the first five years of data taking for the $\mu^{+}$polarity further five years for the opposite polarity in the iron detector, only. We have not included a further five year operational time for the emulsion detector to take into account the mass decrease due to the brick removal in the first five year period $]^{8}$. Notice, however, that a quite relevant improvement with respect to the one-polarity two-detector types case (lower left) is achieved in the $\bar{\delta}$ reconstruction error. More important, an improvement with respect to the two-polarities one-detector type (upper right) can also be observed. In particular, the "clone" regions have completely disappeared, due to the combination of "silver" and "golden" muons with a different $\delta$-dependent oscillation probability. The effect of the

\footnotetext{
${ }^{8}$ This is a quite conservative assumption, being the expected number of bricks to be removed looking for "silver" muons smaller than in the case of $\nu_{\mu} \rightarrow \nu_{\tau}$ oscillations considered in the OPERA proposal, [74.
} 
inclusion of "silver" muons can be seen in Fig. 12, where we present the two-polarities two-detector types combination (Fig. 11, lower right) compared with the same combination but with only a "golden" muon signal. In this figure we can clearly see that "clone" regions are still present and that the near emulsion detector is too small to compete with a 40 Kton iron detector located at the same distance down the neutrino source.
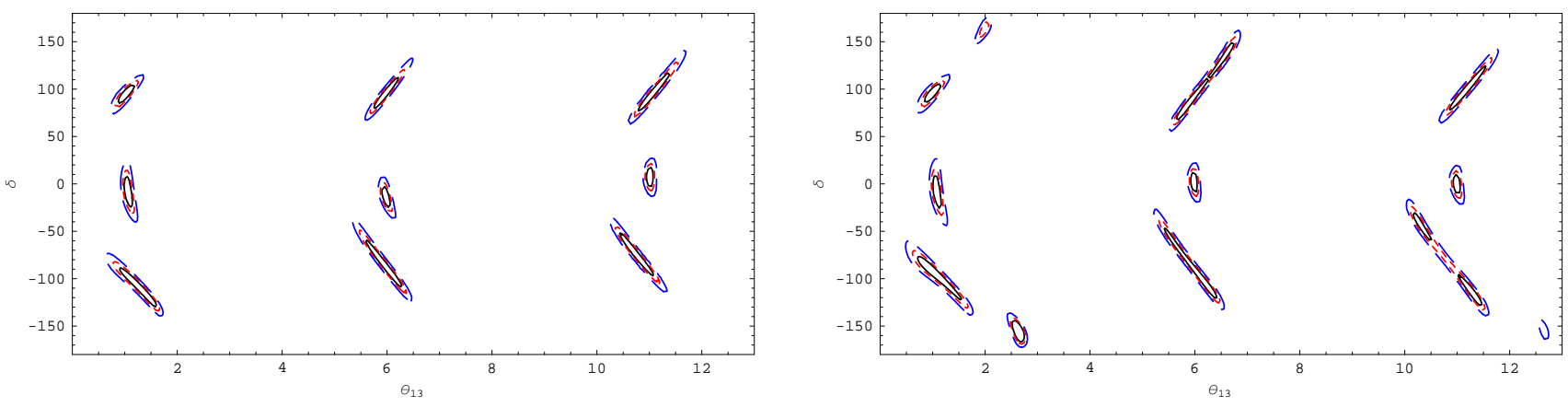

Figure 12: 68.5, 90 and $99 \%$ C.L. contours resulting from a $\chi^{2}$ fit of $\theta_{13}$ and $\delta$, for $\bar{\theta}_{13}=1^{\circ}, 6^{\circ}$ and $11^{\circ}$, and $\bar{\delta}=-90^{\circ}, 0^{\circ}$ and $90^{\circ}$, for the combination of an iron detector at $L=3000 \mathrm{Km}$ and an emulsion detector at $L=732 \mathrm{Km}$ : (left) both "silver" and "golden" muon events are taken into account (same figure as in Fig. 11, lower right); (right) only "golden" muon events are taken into account. Five years of data taking for both polarities in the distant detector and only five years in the $\mu^{+}$polarity in the near detector have been considered.

We also studied the effect of a change in the longest baseline, moving the iron detector from $L=3000 \mathrm{Km}$ to $L=2000 \mathrm{Km}$, with no significant improvement. This was to be expected, since the former distance, $L=3000 \mathrm{Km}$ has been found to be the optimal one to measure the CP-violating phase for the considered set-up, [40]- [50].

We remind here that in 63] it was shown that the optimal combination of two magnetized iron detectors is achieved for $L=3000 \mathrm{Km}$ and $L=7332 \mathrm{Km}$ : in that case, no "clone" region is present (see Fig. 6 of 63]). We prefer however to compare our results for the OPERA-like near detector and magnetized iron not-so-far detector option with the two magnetized iron detectors combination at the same distances.

Up to this moment, we considered an ideal lead-emulsion detector with identification efficiency equal to 1 and no backgrounds. We should consider instead a real detector with a refined analysis of efficiencies and background for the two "wrong-sign muons" signals, $\nu_{e} \rightarrow \nu_{\mu}$ and $\nu_{e} \rightarrow \nu_{\tau}$. A detailed simulation of the expected performance at an OPERA-like detector is under way [75]. However, a preliminary study has been done following the outline of the OPERA proposal [74 and of the recent progress report [76.

We first consider "golden" muons. In this case, it seems reasonable to consider 
a $90 \%$ average efficiency in the neutrino energy range $E_{\nu} \in[5,50] \mathrm{GeV}$. The most relevant background to this channel are "right-sign" muons with a wrong charge assignment: with no improvement with respect to the present OPERA proposal, a level of $10^{-3}$ of charge misidentification is achieved (a level of $10^{-6}$ was envisaged for the magnetized iron detector, see [66]). On the other side, the dominant background at the magnetized iron detector coming from charmed mesons decay with no "right-sign" muon identification is here under control by looking for the decay vertex.

Regarding "silver" muons, we have an unavoidable important reduction in the average efficiency, mainly due to the detector design: the $\tau$ decay vertex cannot be observed if it occurs inside the lead plates. An average efficiency for "silver" muons of $25 \%$ only is achieved in the neutrino energy range $E_{\nu} \in[5,50] \mathrm{GeV}$, [74]. In the case of "sil-

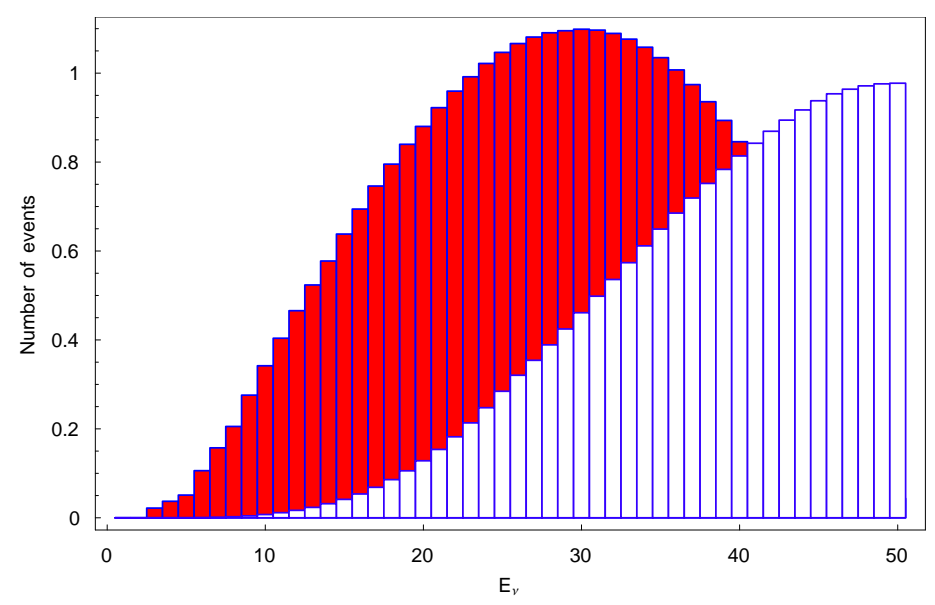

Figure 13: Charmed mesons decay background and "silver" muons signal (shaded) for $\bar{\theta}_{13}=11^{\circ}$ and $\bar{\delta}=90^{\circ}$ in the OPERA-like emulsion detector as a function of the reconstructed neutrino energy (for 1 GeV energy bins).

ver" muon events, the dominant background is the standard charmed mesons decay with no "right-sign" muon identification. Sub-dominant backgrounds coming from $\pi$ and $K$ mesons decay can be kept at a reasonable level by imposing kinematical cuts as described in [74. As an illustrative example, in Fig. 13 we present the charmed mesons decay background and the "silver" muon signal for $\bar{\theta}_{13}=11^{\circ}$ and $\bar{\delta}=90^{\circ}$ as a function of the neutrino energy (for $1 \mathrm{GeV}$ energy bins). Notice how the "silver" muons signal peaks at lower energy with respect to the background, thus allowing us to use the $\nu_{e} \rightarrow \nu_{\tau}$ oscillation to improve the $\left(\theta_{13}, \delta\right)$ reconstruction.

Eventually, we present in Fig. 14 the results for the combination of an iron detector at $L=3000 \mathrm{Km}$ and of an emulsion detector at $L=732 \mathrm{Km}$ with reasonable estimates for the reconstruction efficiencies for "golden" and "silver" muons and for the corresponding backgrounds. We can see that the results obtained with the two-detector 
types combination strongly resembles those obtained with the reference two iron detectors set-up (Fig. 11, upper right). In particular, we notice a general improvement at the $68 \%$ C.L. but we still observe "clone" regions for small values of $\bar{\theta}_{13}$.

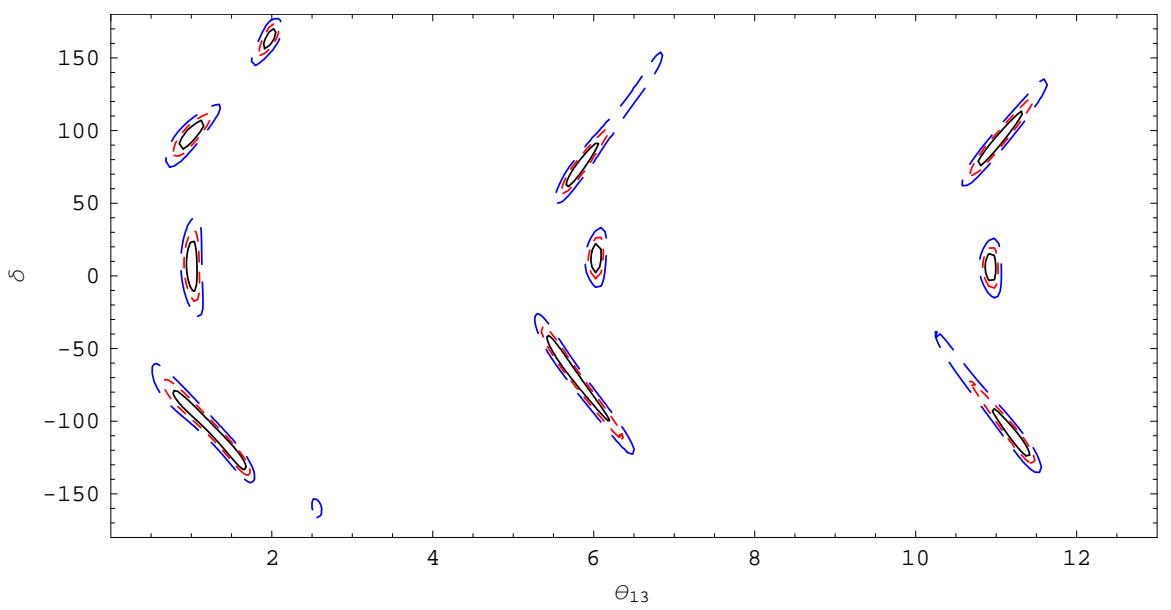

Figure 14: $\quad 68.5,90$ and $99 \%$ C.L. contours resulting from a $\chi^{2}$ fit of $\theta_{13}$ and $\delta$, for $\bar{\theta}_{13}=1^{\circ}, 6^{\circ}$ and $11^{\circ}$, and $\bar{\delta}=-90^{\circ}, 0^{\circ}$ and $90^{\circ}$, for the combination of an iron detector at $L=3000 \mathrm{Km}$ (with "golden" muon events) and an emulsion detector at $L=732$ Km (with "golden" and "silver" muon events). Realistic estimates for efficiencies and backgrounds at both the iron and the emulsion detector have been taken into account. Five years of data taking for both polarities in the distant detector and only five years in the $\mu^{+}$polarity in the near detector have been considered.

Although the results of Fig. 14 do not indicate a strong improvement with respect to the reference two iron detectors set-up, two comments are in order: first, we have been considering up to now an OPERA-like detector with the characteristics of the detector described in [74 which is currently under construction and should be operational by year 2006. We therefore study the effects on the physics reach of foreseeable improvements in the following. Secondly, it seems to us that the combination of two different detector types with different characteristics and systematics could be of the utmost importance in the project of a facility for precision measurements of the leptonic mixing matrix such as the Neutrino Factory.

In Tab. 1 we schematically present which are the main advantages of an increase in the emulsion detector mass, or of a reduction of the main backgrounds, considering the presence or absence of "clone" regions and the maximal error in $\delta$ that can be achieved. The main background for "silver" muon events $B_{\text {silver }}$ (originating from charmed mesons decay) is normalized to the value that can be deduced on the basis of the OPERA proposal. In this case we do not consider that significant improvements can be achieved and we restrict ourselves to a 10 to $20 \%$ reduction. We believe that, on the other hand, the main background for "golden" muon events $B_{\text {golden }}$ (originating 


\begin{tabular}{|c|c|c|c|c|}
\hline Mass (Kton) & $B_{\text {silver }}$ & $B_{\text {golden }}$ & Clone regions & $\delta_{\min }, \delta_{\max }$ at $99 \%$ C.L. \\
\hline \multirow{6}{*}{2} & 1.0 & $10^{-3}$ & At $68 \%$ C.L. & - \\
\hline & 0.9 & $10^{-3}$ & At $68 \%$ C.L. & - \\
\hline & 0.8 & $10^{-3}$ & At $68 \%$ C.L. & - \\
\hline & 1.0 & $10^{-4}$ & At $68 \%$ C.L. & - \\
\hline & 0.9 & $10^{-4}$ & At $90 \%$ C.L. & - \\
\hline & 0.8 & $10^{-4}$ & At $90 \%$ C.L. & - \\
\hline \multirow{6}{*}{3} & 1.0 & $10^{-3}$ & At $99 \%$ C.L. & - \\
\hline & 0.9 & $10^{-3}$ & At $99 \%$ C.L. & - \\
\hline & 0.8 & $10^{-3}$ & At $99 \%$ C.L. & - \\
\hline & 1.0 & $10^{-4}$ & No & $50^{\circ}-100^{\circ}$ \\
\hline & 0.9 & $10^{-4}$ & No & $50^{\circ}-110^{\circ}$ \\
\hline & 0.8 & $10^{-4}$ & No & $50^{\circ}-110^{\circ}$ \\
\hline \multirow{6}{*}{4} & 1.0 & $10^{-3}$ & $\mathrm{No}$ & $55^{\circ}-105^{\circ}$ \\
\hline & 0.9 & $10^{-3}$ & No & $55^{\circ}-105^{\circ}$ \\
\hline & 0.8 & $10^{-3}$ & No & $55^{\circ}-105^{\circ}$ \\
\hline & 1.0 & $10^{-4}$ & No & $60^{\circ}-110^{\circ}$ \\
\hline & 0.9 & $10^{-4}$ & No & $60^{\circ}-110^{\circ}$ \\
\hline & 0.8 & $10^{-4}$ & No & $70^{\circ}-110^{\circ}$ \\
\hline
\end{tabular}

Table 1: Effects of a mass increase or of a "silver" ( $\left.B_{\text {silver }}\right)$ or "golden" ( $\left.B_{\text {golden }}\right)$ main background reduction for the emulsion detector, for $\bar{\theta}_{13}=1^{\circ}$ and $\bar{\delta}=90^{\circ}$. Whenever a "clone" region is present, no overall error on $\delta$ is given.

from charge misidentification) could be significantly reduced: we therefore present results for a $10^{-3}$ (the predicted level in the OPERA proposal) and for a $10^{-4}$ charge misidentification. Finally, having in mind that in the last years the scanning power increased by about a factor 10 every two years, we consider as realistic the assumption that, by the time the Neutrino factory will become operational, we will be able to scan a larger number of events, say a factor two. Therefore, we studied the physical reach of the Neutrino factory-detectors set-up as a function of the lead-emulsion mass, considering a linear increase in the mass from 2 Kton to 4 Kton.

The outcome of this analysis is the following:

- "Clone" regions disappear increasing the emulsion mass. For a 4 Kton detector no ambiguity is present. An improvement in the charge identification (i.e., a reduction of "golden" muons main background) implies generally a decrease in the statistical significance of "clone" regions.

- When no "clone" regions are observed, a reduction of "golden" muons main background induces a shift in the $99 \%$ C.L. allowed region for $\delta$ towards higher values. In the optimal case we observe a gaussian-distributed region with $\Delta \delta=$ $\pm 20^{\circ}$. 
- A 10 to $20 \%$ reduction of the "silver" muons main background (charmed mesons decay) does not seem to improve significantly the previous results.

We remind that this table should be interpreted as an indication of which kind of improvement in the emulsion detector helps more to improve the physics reach of the envisaged two detector types combination.

\section{Conclusions}

It was previously shown 40] that the "golden" wrong-sign muon signal at the Neutrino Factory can be extremely useful to measure simultaneously two of the parameters of the PMNS leptonic mixing matrix, $\theta_{13}$ and $\delta$. In [63] was first noticed that degenerate regions in the $\left(\theta_{13}, \delta\right)$ parameter space occur in many cases, severely reducing the Neutrino Factory sensitivity to the CP-violating phase $\delta$.

In the first part of the paper we describe how the $\left(\theta_{13}, \delta\right)$ ambiguity arises in $\nu_{e} \rightarrow \nu_{\mu}$ oscillation due to the equiprobability curves in the $\left(\theta_{13}, \delta\right)$ plane at fixed neutrino energy. We then extend our analysis showing how the same phenomenon can be observed in a real experiment: equal-number-of-events (ENE) curves for any given neutrino energy bin appears and their intersections in the $\left(\theta_{13}, \delta\right)$ plane explain how and where do "clone" regions arise. Our analysis is subsequently compared with the results of simulations with a realistic magnetized iron detector, already studied in previous papers.

In the second part of the paper, we propose the use of $\nu_{e} \rightarrow \nu_{\tau}$ oscillation to solve the $\left(\theta_{13}, \delta\right)$ ambiguity problem. We apply the arguments presented in the case of $\nu_{e} \rightarrow \nu_{\mu}$ and we show how $\nu_{e} \rightarrow \nu_{\tau}$ actually give a complementary information that can be used to improve the measurement of the phase $\delta$. However, to take full advantage of this "silver" channel we should use a detector of a different kind to distinguish muons originated from $\tau$ decay from the so-called "golden" muons. In the last part of the paper we therefore perform simulations using the combination of a realistic magnetized iron detector at $L=3000 \mathrm{Km}$ and of an OPERA-like lead-emulsion detector located at $L=732 \mathrm{Km}$ from the Neutrino Factory. The $\tau$ decay vertex recognition in the emulsion detector allow us to separate "silver" from "golden" muon events and to strongly reduce the effect of the charmed mesons decay background.

We first use an ideal lead-emulsion detector with the mass currently envisaged by the OPERA Collaboration (2 Kton), perfect reconstruction efficiency and no background, showing how the "clone" regions disappear for any value of $\theta_{13} \geq 1^{\circ}$. Eventually, we consider a realistic estimate of the reconstruction efficiency and of the main backgrounds both for "golden" and "silver" muon events at the emulsion detector, following the outline of the OPERA proposal [74]. In this case, "clone" regions for $\theta_{13} \simeq 1^{\circ}$ do appear and our results are similar to those that we obtain for a combination of two realistic magnetized iron detectors with $L=732$ and $L=3000$ baselines. However, we stress here that the OPERA-like 2 Kton lead-emulsion detector is under construction 
and should be operational by mid 2006 to exploit the CNGS beam. We can therefore study how possible improvements of the detector (from 2006 till the time the Neutrino factory will become operational) affect the results of our analysis. In particular we remind that a moderate scaling in the lead-emulsion detector mass could indeed be seriously taken into account (due to the rapid increase in the emulsion scanning power). The outcome of this study is that an increase in the emulsion mass from 2 to 4 Kton eliminates completely the "clone" regions for $\theta_{13} \geq 1^{\circ}$. On the other hand, a moderate increase in the main background rejection does not seem to improve in a significant way the previous results.

As a final comment we believe that the two-detector types combination should be investigated further, even in the case of results comparable to those of a two-baselines magnetized iron detector combination, since having detectors with different characteristics and systematics can be extremely helpful to ameliorate the $\delta$ measurement.

\section{Acknowledgements}

We are deeply indebted with Paolo Lipari and Maurizio Lusignoli for exhaustive discussions on many different aspects of this paper. We acknowledge useful discussions with L. Di Lella, M.B. Gavela, J.J. Gomez-Cadenas, P. Hernandez, L. Ludovici, Carlos Peña-Garay, S. Rigolin, G. Rosa, P. Strolin, D. Zardetto and P. Zucchelli. 


\section{A Perturbative expansion in $\Delta \theta$}

If $\theta_{13}$ is large enough, we can expand eqs. (5) and (6) in powers of $\Delta \theta$ for any value of $\Delta \theta$ in the allowed region of Fig. [1.

At first order in $\Delta \theta$,

$$
\left\{\begin{array}{l}
f\left(\theta_{13}, \bar{\theta}_{13}\right) \simeq-\Delta \theta \tilde{f}\left(\bar{\theta}_{13}\right)+\mathcal{O}\left(\Delta \theta^{2}\right) \simeq-4 \Delta \theta \frac{\cos \left(2 \bar{\theta}_{13}\right)}{\sin \left(\theta_{13}\right)}+\mathcal{O}\left(\Delta \theta^{2}\right), \\
g\left(\theta_{13}, \bar{\theta}_{13}\right) \simeq 1-\Delta \theta \tilde{g}\left(\bar{\theta}_{13}\right)+\mathcal{O}\left(\Delta \theta^{2}\right) \simeq 1-\Delta \theta \frac{3 \cos \left(2 \bar{\theta}_{13}\right)-1}{\sin \left(2 \theta_{13}\right)}+\mathcal{O}\left(\Delta \theta^{2}\right),
\end{array}\right.
$$

where, for $\bar{\theta}_{13} \in\left[0^{\circ}, 13^{\circ}\right], \tilde{f}$ and $\tilde{g}$ are positive functions of $\bar{\theta}_{13}$.

For neutrinos we get

$$
\delta_{+}=\frac{\Delta_{a t m} L}{2} \pm\left\{\left(\bar{\delta}-\frac{\Delta_{a t m} L}{2}\right)-\Delta \theta \frac{4 R_{+} \tilde{f}\left(\bar{\theta}_{13}\right)+\cos \left(\bar{\delta}-\frac{\Delta_{a t m} L}{2}\right) \tilde{g}\left(\bar{\theta}_{13}\right)}{\left|\sin \left(\bar{\delta}-\frac{\Delta_{a t m} L}{2}\right)\right|}\right\}
$$

and for antineutrinos we get

$$
\delta_{-}=-\frac{\Delta_{a t m} L}{2} \pm\left\{\left(\bar{\delta}+\frac{\Delta_{a t m} L}{2}\right)-\Delta \theta \frac{4 R_{-} \tilde{f}\left(\bar{\theta}_{13}\right)+\cos \left(\bar{\delta}+\frac{\Delta_{a t m} L}{2}\right) \tilde{g}\left(\bar{\theta}_{13}\right)}{\left|\sin \left(\bar{\delta}+\frac{\Delta_{a t m} L}{2}\right)\right|}\right\} .
$$

Notice that from eq. (55), for $\Delta \theta=0$, we get $\delta_{ \pm}=\bar{\delta}$ or $\delta_{ \pm}=-\bar{\delta} \pm \Delta_{a t m} L$. For $\Delta \theta \neq 0$, we get the two branches of the equiprobability curves.

If we turn on the $\Delta \theta$ correction at first order, the results of Fig. 2 are easily understood with the following argument: consider two different equiprobability curves for neutrinos, for two different values of the neutrino energy $E_{1}$ and $E_{2}, E_{2} \geq E_{1}$. The intersections between the two curves are defined by

$$
\delta_{+}\left(E_{1}\right)=\delta_{+}\left(E_{2}\right) .
$$

Solving for $\Delta \theta$ we get two solutions:

$$
\left\{\begin{array}{l}
\Delta \theta=0 \\
\Delta \theta=L \frac{\Delta_{a t m}\left(E_{1}\right)-\Delta_{a t m}\left(E_{2}\right)}{H\left(E_{2}\right)-H\left(E_{1}\right)}
\end{array}\right.
$$

where

$$
H(E)=\frac{4 R_{+}(E) \tilde{f}\left(\bar{\theta}_{13}\right)+\cos \left(\bar{\delta}+\frac{\Delta_{a t m}(E) L}{2}\right) \tilde{g}\left(\bar{\theta}_{13}\right)}{\left|\sin \left(\bar{\delta}+\frac{\Delta_{a t m}(E) L}{2}\right)\right|} .
$$


For $\Delta \theta=0$, we get $\delta=\bar{\delta}$. On the other hand, the second intersection gets an $\mathcal{O}(\Delta \theta)$ correction:

$$
\delta=-\bar{\delta}+L \Delta_{a t m}\left(E_{1}\right)+\Delta \theta H\left(E_{1}\right) .
$$

Being $H(E)$ and (for the considered parameters and baselines) the second solution for $\Delta \theta$ both positive quantities, the second intersection between any two equiprobability curves for neutrinos will be displaced towards negative values of $\delta$ and positive values of $\Delta \theta$. This is precisely what observed in the upper row of Fig. 2. For the considered set of input parameters, baselines and neutrino energy range the same argument applies to the antineutrino equiprobability curves also, as it can be seen in the lower row of Fig. 2.

\section{B Formulae for $\tau$ decay and $\nu_{\tau} N$ cross-section}

Grouping events in bins of the final muon energy $E_{\mu}$, with the size of the energy bin depending on the energy resolution $\Delta E_{\mu}$ of the considered detector, the number of "silver" muon events in the i-th energy bin for the input pair $\left(\bar{\theta}_{13}, \bar{\delta}\right)$ and for a parent muon energy $\bar{E}_{\mu}$ is:

$$
\begin{aligned}
N_{\mu^{\mp}}^{s}\left(\bar{\theta}_{13}, \bar{\delta}\right)=B R(\tau & \rightarrow \mu)\left\{\left[\frac{d N_{\mu^{\mp}}\left(E_{\mu}, E_{\tau}\right)}{d E_{\mu}} \otimes \frac{d \sigma_{\nu_{\tau}\left(\bar{\nu}_{\tau}\right)}\left(E_{\tau}, E_{\nu}\right)}{d E_{\tau}}\right]\right. \\
& \left.\otimes P_{e \tau}^{ \pm}\left(E_{\nu}, \bar{\theta}_{13}, \bar{\delta}\right) \otimes \frac{d \Phi_{\nu_{e}\left(\bar{\nu}_{e}\right)}\left(E_{\nu}, \bar{E}_{\mu}\right)}{d E_{\nu}}\right\}_{E_{i}}^{E_{i}+\Delta E_{\mu}},
\end{aligned}
$$

where $\otimes$ stands for a convolution integral on the intermediate energy.

Several comments are in order:

- The differential neutrino flux $d \Phi_{\nu_{e}\left(\bar{\nu}_{e}\right)}\left(E_{\nu}, \bar{E}_{\mu}\right) / d E_{\nu}$ has been given in eq. (10).

- The $\nu_{e} \rightarrow \nu_{\tau}$ oscillation probability has been given in eq. (20).

- The $\nu_{\tau} N$ cross-section

The (anti)neutrino-nucleon differential cross sections on an isoscalar target, defined as the average of the differential cross-sections on proton and neutron, can be divided in three components: the elastic, the quasi-elastic and the DIS crosssection. We refer to [77] for a detailed discussion on the different contributions to these components. The DIS cross-section can be expressed in terms of the isoscalar structure functions $F_{i}(x)(i=1, \ldots, 5)$ :

$$
\begin{aligned}
\frac{d^{2} \sigma^{\nu(\bar{\nu})}}{d x d y} & =\frac{G_{F}^{2} M E_{\nu}}{\pi}\left\{y\left(x y+\frac{m_{l}^{2}}{2 M E_{\nu}}\right) F_{1}(x)+\left(1-y-\frac{M x y}{2 E_{\nu}}-\frac{m_{l}^{2}}{4 E_{\nu}^{2}}\right) F_{2}(x)\right. \\
& \pm\left[x y\left(1-\frac{y}{2}\right)-y \frac{m_{l}^{2}}{4 M E_{\nu}}\right] F_{3}(x)+\left(x y \frac{m_{l}^{2}}{2 M E_{\nu}}+\frac{m_{l}^{4}}{4 M^{2} E_{\nu}^{2}}\right) F_{4}(x)
\end{aligned}
$$




$$
\left.-\frac{m_{l}^{2}}{2 M E_{\nu}} F_{5}(x)\right\}
$$

where $M$ is the nucleon mass and $m_{l}$ the charged lepton mass; $x=Q^{2} / 2 M E_{\nu} y$ is the DIS Bjorken variable and $y=1-E_{l} / E_{\nu}$ depends on the fraction of neutrino energy carried away by the charged lepton. Notice that the $x, y$ variables defined in this Appendix should be interpreted as local variables: outside the Appendix the same letters may represent different objects (we are simply following the standard notation for the DIS cross-section).

Making use of the Callan-Gross relation, $2 x F_{1}(x)=F_{2}(x)$, and of the AlbrightJarlskog relations [73, $F_{4}(x)=0, x F_{5}(x)=F_{2}(x)$, eq. (29) depends on two independent structure functions, only. Using the quark parton model (see [78] and refs. therein, for example) the isoscalar structure functions $F_{2}(x)$ and $x F_{3}(x)$ are:

$$
\left\{\begin{array}{l}
F_{2}^{\nu}(x)=x[(u+d+2 s)+(\bar{u}+\bar{d}+2 \bar{c})], \\
F_{2}^{\bar{\nu}}(x)=x[(u+d+2 c)+(\bar{u}+\bar{d}+2 \bar{s})], \\
x F_{3}^{\nu}(x)=x[(u+d+2 s)-(\bar{u}+\bar{d}+2 \bar{c})], \\
x F_{3}^{\bar{\nu}}(x)=x[(u+d+2 c)-(\bar{u}+\bar{d}+2 \bar{s})] .
\end{array}\right.
$$

Eventually, the structure functions, given in terms of the parton distribution at a definite value of $Q^{2}$ [79], must be evolved according to the DGLAP equations to compute the $\nu_{\tau} N$ cross-section for a given neutrino energy.

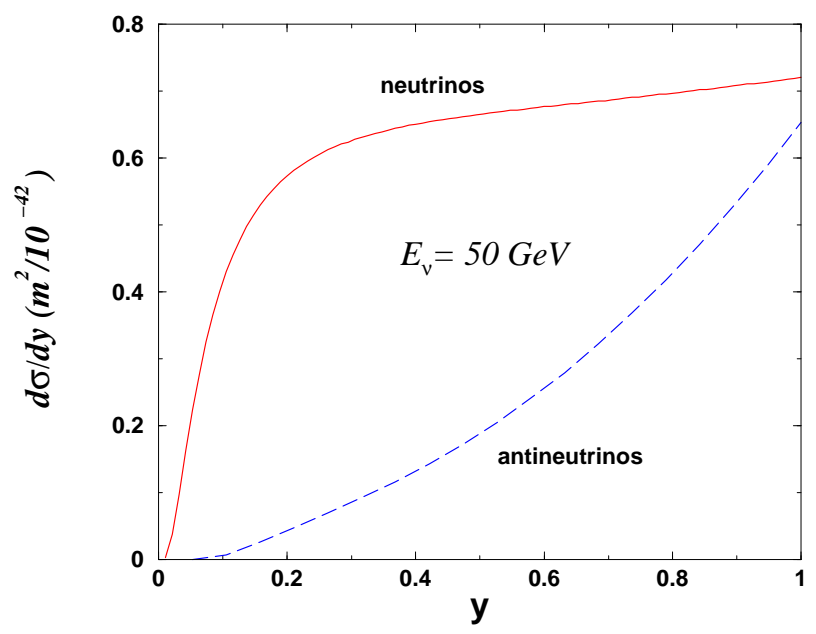

Figure 15: Neutrino and antineutrino - nucleon differential cross section as a function of $y$ for $E_{\nu}=\bar{E}_{\mu}$.

In Fig. 15 we show the dependence of the $d \sigma / d y$ on $y$ for a fixed value of $E_{\nu}$ (in this case, $E_{\mu}=\bar{E}_{\mu}$ ). Notice the strong suppression of the antineutrino cross-section 
with respect to the neutrino one for high and intermediate $\tau$ energy $(y \rightarrow 0)$. The integration limits for the $(x, y)$ variables are reported in Fig. 16 for different neutrino energies [80].

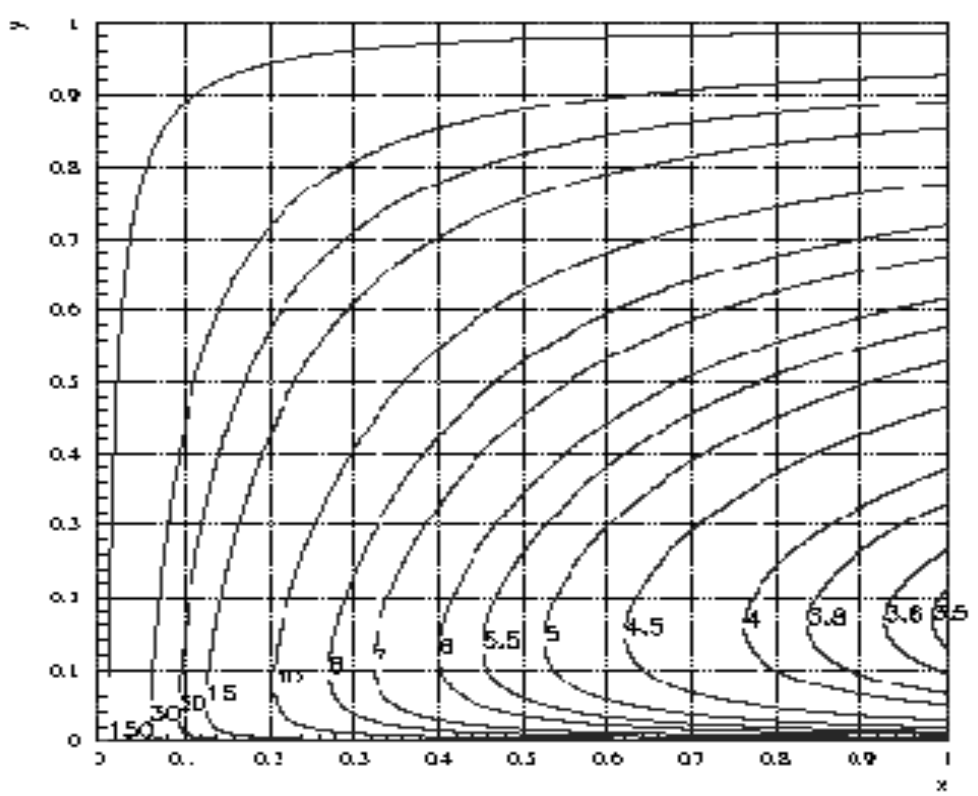

Figure 16: Kinematical bounds in the $x, y$ plane for different neutrino energies, 80].

\section{- The $\tau$ decay rate}

The muon distribution is given, in the $\tau$ rest frame, by the following expression:

$$
\frac{d^{2} N_{\mu^{ \pm}}}{d x^{\prime} d \cos \theta}=\frac{1}{2}\left[f_{0}\left(x^{\prime}, R\right) \mp \mathcal{P}_{\tau} f_{1}\left(x^{\prime}, R\right) \cos \theta\right]
$$

where $R=m_{\mu} / m_{\tau}, \mathcal{P}_{\tau}$ is the average $\tau$ polarization along the $\tau$ direction in the laboratory frame, $x^{\prime}=2 E_{\nu} / m_{\tau}$ (not to be confused with the Bjorken $x$ variable defined for the cross-section) and $\theta$ is the angle between the muon momentum vector and the $\tau$ spin direction. The two $f$-functions are:

$$
\begin{aligned}
& f_{0}\left(x^{\prime}, R\right)=2 \sqrt{x^{\prime 2}-4 R^{2}}\left[-4 R^{2}+3\left(1+R^{2}\right) x^{\prime}-2 x^{\prime 2}\right] \\
& f_{1}\left(x^{\prime}, R\right)=2\left(x^{\prime 2}-4 R^{2}\right)\left[1+3 R^{2}-2 x^{\prime}\right]
\end{aligned}
$$

and they reduce to the standard $f_{0}\left(x^{\prime}\right)$ and $f_{1}\left(x^{\prime}\right)$ functions for the $\nu_{\mu}$ and $e$ flux in the $R \rightarrow 0$ limit 81. The boost in the laboratory frame is given by the 
following relations:

$$
\begin{aligned}
y^{\prime} & =\frac{x^{\prime}}{2}+\frac{\beta}{2}\left[x^{\prime 2}-4 R^{2}\right]^{1 / 2} \cos \theta \\
\cos \theta^{\prime} & =\frac{\beta x^{\prime}+\left(x^{\prime 2}-4 R^{2}\right)^{1 / 2} \cos \theta}{\left[\left(x^{\prime}+\beta\left(x^{\prime 2}-4 R^{2}\right)^{1 / 2} \cos \theta\right)-4\left(1-\beta^{2}\right) R^{2}\right]^{1 / 2}}
\end{aligned}
$$

where $y^{\prime}=E_{\mu} / E_{\tau}$ and $\theta^{\prime}$ is the angle boosted in the lab frame.

\section{References}

[1] Y. Fukuda et al. [Kamiokande Collaboration], Phys. Lett. B335 (1994) 237.

[2] R. Becker-Szendy et al., Nucl. Phys. Proc. Suppl. 38 (1995) 331.

[3] Y. Fukuda et al. [SuperKamiokande Collaboration], Phys. Rev. Lett. 82 (1999) 2644.

[4] W. W. Allison et al. [Soudan-2 Collaboration], Phys. Lett. B449 (1999) 137.

[5] M. Ambrosio et al. [MACRO Collaboration], Phys. Lett. B434 (1998) 451.

[6] T. Toshito [SuperKamiokande Collaboration], arXiv:hep-ex/0105023.

[7] B. T. Cleveland et al., Astrophys. J. 496 (1998) 505.

[8] Y. Fukuda et al. [Kamiokande Collaboration], Phys. Rev. Lett. 77 (1996) 1683.

[9] W. Hampel et al. [GALLEX Collaboration], Phys. Lett. B447 (1999) 127.

[10] J. N. Abdurashitov et al. [SAGE Collaboration], Phys. Rev. C60 (1999) 055801.

[11] Y. Suzuki [Super-Kamiokande Collaboration], Nucl. Phys. Proc. Suppl. 77 (1999) 35.

[12] Q. R. Ahmad et al. [SNO Collaboration], Phys. Rev. Lett. 87 (2001) 071301 arXiv:nucl-ex/0106015.

[13] Q. R. Ahmad et al. [SNO Collaboration], arXiv:nucl-ex/0204008.

[14] Q. R. Ahmad et al. [SNO Collaboration], arXiv:nucl-ex/0204009.

[15] B. Pontecorvo, Sov. Phys. JETP 6 (1957) 429 [Zh. Eksp. Teor. Fiz. 33 (1957) 549].

[16] Z. Maki, M. Nakagawa and S. Sakata, Prog. Theor. Phys. 28 (1962) 870. 
[17] B. Pontecorvo, Sov. Phys. JETP 26 (1968) 984.

[18] V. N. Gribov and B. Pontecorvo, Phys. Lett. B 28 (1969) 493.

[19] Particle Data Book, Eur. Phys. J. C 15 (2000) 1.

[20] G. L. Fogli, E. Lisi and A. Marrone, Phys. Rev. D 65 (2002) 073028 arXiv:hepph/0110089; Phys. Rev. D 64 (2001) 093005 arXiv:hep-ph/0105139.

[21] L. Wolfenstein, Phys. Rev. D 17 (1978) 2369; Phys. Rev. D 20 (1979) 2634; S.P. Mikheyev and A. Yu. Smirnov, Sov. J. Nucl. Phys. 42 (1986) 913.

[22] V. Barger, D. Marfatia, K. Whisnant and B. P. Wood, arXiv:hep-ph/0204253.

[23] J. N. Bahcall, M. C. Gonzalez-Garcia and C. Pena-Garay, arXiv:hep-ph/0204314.

[24] A. Bandyopadhyay, S. Choubey, S. Goswami and D. P. Roy, arXiv:hep$\mathrm{ph} / 0204286$.

[25] P. C. Holanda and A. Y. Smirnov, arXiv:hep-ph/0205241.

[26] C. Athanassopoulos et al. [LSND Collaboration], Phys. Rev. Lett. 81 (1998) 1774.

[27] A. Aguilar et al. [LSND Collaboration], Phys. Rev. D 64 (2001) 112007 arXiv:hep-ex/0104049.

[28] J. Kleinfeller [KARMEN Collaboration], Nucl. Phys. Proc. Suppl. 87 (2000) 281.

[29] E. Church et al. [BooNe Collaboration], nucl-ex/9706011.

[30] M. Sorel, arXiv:hep-ph/0205207.

[31] A. Donini, M. B. Gavela, P. Hernandez and S. Rigolin, Nucl. Phys. 574 (2000) 23; Nucl. Instrum. Meth. A 451 (2000) 58 hep-ph/9910516]; arXiv:hep-ph/0007283.

[32] A. Donini and D. Meloni, Eur. Phys. J. C 22 (2001) 179 arXiv:hep-ph/0105089; arXiv:hep-ph/0105163; arXiv:hep-ph/0107274.

[33] A. Donini, M. Lusignoli and D. Meloni, Nucl. Phys. B 624, 405 (2002) arXiv:hepph/0107231]; D. Meloni, arXiv:hep-ph/0204351.

[34] M. Apollonio et al., Phys. Lett. B 338 (1998) 383.

[35] S. Geer, Phys. Rev. D 57 (1998) 6989 [Erratum-ibid. D 59 (1998) 039903] thepph/9712290.

[36] A. De Rujula, M. B. Gavela and P. Hernandez, Nucl. Phys. B 547 (1999) 21 arXiv:hep-ph/9811390. 
[37] K. Dick, M. Freund, M. Lindner and A. Romanino, Nucl. Phys. B 562 (1999) 29 hep-ph/9903308.

[38] V. Barger, S. Geer and K. Whisnant, Phys. Rev. D 61 (2000) 053004 hep$\mathrm{ph} / 9906487$.

[39] A. Bueno, M. Campanelli and A. Rubbia, Nucl. Phys. B 573 (2000) 27.

[40] A. Cervera et al., Nucl. Phys. B 579 (2000) 17 [Erratum-ibid. B 593 (2001) 731] arXiv:hep-ph/0002108.

[41] C. Albright et al., hep-ex/0008064.

[42] A. Blondel et al., Nucl. Instrum. Meth. A 451 (2000) 102.

[43] T. Adams et al., in Proc. of the APS/DPF/DPB Summer Study on the Future of Particle Physics (Snowmass 2001) ed. R. Davidson and C. Quigg, arXiv:hep$\mathrm{ph} / 0111030$.

[44] M. Koike and J. Sato, Phys. Rev. D 61 (2000) 073012 [Erratum-ibid. D 62 (2000) 079903] [arXiv:hep-ph/9909469]; T. Ota and J. Sato, Phys. Rev. D 63 (2001) 093004 arXiv:hep-ph/0011234; M. Koike, T. Ota and J. Sato, Phys. Rev. D 65 (2002) 053015 arXiv:hep-ph/0011387; T. Ota, J. Sato and Y. Kuno, Phys. Lett. B 520 (2001) 289 arXiv:hep-ph/0107007.

[45] M. Freund, M. Lindner, S. T. Petcov and A. Romanino, Nucl. Instrum. Meth. A 451 (2000) 18; M. Freund, P. Huber and M. Lindner, Nucl. Phys. B 585 (2000) 105 arXiv:hep-ph/0004085.

[46] M. Freund, P. Huber and M. Lindner, Nucl. Phys. B 615 (2001) 331 arXiv:hep$\mathrm{ph} / 0105071$.

[47] V. Barger, S. Geer, R. Raja and K. Whisnant, Phys. Rev. D 62 (2000) 073002 hep-ph/0003184; Phys. Lett. B 485 (2000) 379 arXiv:hep-ph/0004208; Phys. Rev. D 63 (2001) 033002 arXiv:hep-ph/0007181.

[48] A. Bueno, M. Campanelli and A. Rubbia, Nucl. Phys. B 589 (2000) 577 hep$\mathrm{ph} / 0005007$.

[49] M. Campanelli, A. Bueno and A. Rubbia, Nucl. Instrum. Meth. A 451 (2000) 207.

[50] A. Rubbia, hep-ph/0106088.

[51] A. Bueno, M. Campanelli, S. Navas-Concha and A. Rubbia, arXiv:hep$\mathrm{ph} / 0112297$.

[52] P. Lipari, Phys. Rev. D 64 (2001) 033002 arXiv:hep-ph/0102046]. 
[53] J. Pinney and O. Yasuda, Phys. Rev. D 64 (2001) 093008 arXiv:hepph/0105087; O. Yasuda, arXiv:hep-ph/0203273.

[54] J. J. Gomez-Cadenas and D. A. Harris, FERMILAB-PUB-02-044-T.

[55] V. D. Barger, S. Geer, R. Raja and K. Whisnant, Phys. Rev. D 63 (2001) 113011 arXiv:hep-ph/0012017.

[56] V. Barger et al., hep-ph/0103052.

[57] J. J. Gomez-Cadenas et al. [CERN working group on Super Beams Collaboration], arXiv:hep-ph/0105297.

[58] H. Minakata and H. Nunokawa, Phys. Lett. B 495 (2000) 369 arXiv:hepph/0004114; JHEP 0110 (2001) 001 arXiv:hep-ph/0108085.

[59] K. Dick, M. Freund, P. Huber and M. Lindner, Nucl. Phys. B 598 (2001) 543 arXiv:hep-ph/0008016.

[60] P. Huber, M. Lindner and W. Winter, arXiv:hep-ph/0204352.

[61] P. Zucchelli, arXiv:hep-ex/0107006.

[62] M. Campanelli, arXiv:hep-ex/0204004.

[63] J. Burguet-Castell, M. B. Gavela, J. J. Gomez-Cadenas, P. Hernandez and O. Mena, Nucl. Phys. B 608 (2001) 301 arXiv:hep-ph/0103258; J. BurguetCastell and O. Mena, arXiv:hep-ph/0108109.

[64] T. Kajita, H. Minakata and H. Nunokawa, Phys. Lett. B 528 (2002) 245 arXiv:hep-ph/0112345.

[65] V. Barger, D. Marfatia and K. Whisnant, Phys. Rev. D 65, 073023 (2002) arXiv:hep-ph/0112119.

[66] A. Cervera, F. Dydak and J. Gomez Cadenas, Nucl. Instrum. Meth. A 451 (2000) 123.

[67] M. Freund, Phys. Rev. D 64 (2001) 053003 arXiv:hep-ph/0103300.

[68] K. Kimura, A. Takamura and H. Yokomakura, arXiv:hep-ph/0203099; arXiv:hepph/0205295.

[69] H. Minakata, H. Nunokawa and S. Parke, arXiv:hep-ph/0204171.

[70] A. M. Dziewonski and D.L. Anderson, Phys. Earth Planet. Int. 25 (1981) 297.

[71] A. Broncano and O. Mena, arXiv:hep-ph/0203052. 
[72] See http://vassilo.home.cern.ch/vassilo/NGS/sig_opera.dat

[73] C. H. Albright and C. Jarlskog, Nucl. Phys. B 84 (1975) 467.

[74] M. Guler et al. [OPERA Collaboration], CERN-SPSC-2000-028.

[75] D. Autiero et al., in preparation.

[76] M. Guler et al. [OPERA Collaboration], CERN-SPSC-2001-025.

[77] P. Lipari, M. Lusignoli, D. Meloni and D. Zardetto, in preparation.

[78] E. A. Paschos and J. Y. Yu, Phys. Rev. D 65, 033002 (2002) arXiv:hepph/0107261.

[79] A. D. Martin, R. G. Roberts, W. J. Stirling and R. S. Thorne, Eur. Phys. J. C 23, 73 (2002) arXiv:hep-ph/0110215.

[80] P. Zucchelli, PhD thesis, unpublished.

[81] T. K. Gaisser, Cosmic Rays and Particle Physics, Cambridge University Press, 1990. 